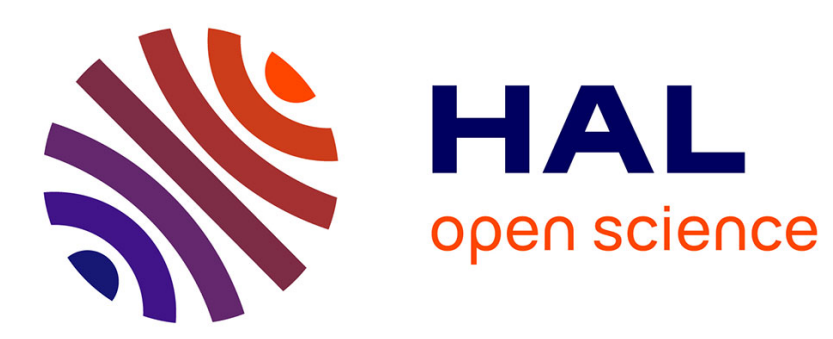

\title{
Generalized Newton's methods for the approximation and resolution of frictional contact problems in elasticity
}

Yves Renard

\section{To cite this version:}

Yves Renard. Generalized Newton's methods for the approximation and resolution of frictional contact problems in elasticity. Computer Methods in Applied Mechanics and Engineering, 2013, 256, pp.38-55. 10.1016/j.cma.2012.12.008 . hal-00722114v3

\section{HAL Id: hal-00722114 \\ https://hal.science/hal-00722114v3}

Submitted on 6 Dec 2012

HAL is a multi-disciplinary open access archive for the deposit and dissemination of scientific research documents, whether they are published or not. The documents may come from teaching and research institutions in France or abroad, or from public or private research centers.
L'archive ouverte pluridisciplinaire HAL, est destinée au dépôt et à la diffusion de documents scientifiques de niveau recherche, publiés ou non, émanant des établissements d'enseignement et de recherche français ou étrangers, des laboratoires publics ou privés. 


\title{
Generalized Newton's methods for the approximation and resolution of frictional contact problems in elasticity
}

\author{
Yves Renard, \\ Université de Lyon, CNRS, \\ INSA-Lyon, ICJ UMR5208, LAMCOS UMR5259, F-69621, Villeurbanne, France, \\ Yves.Renard@insa-lyon.fr.
}

December 6, 2012

\begin{abstract}
In this paper, some new generalized Newton's methods for the resolution of elastostatic frictional contact problem approximated by finite elements are presented and compared to existing ones. A numerical experimentation is performed to compare the different methods, especially with respect to the sensitivity to the method parameter. Two different strategies to approximate the contact and friction condition are considered: a nodal and an integral one. Existence and uniqueness results of the solution to the discretized problem are also discussed.
\end{abstract}

Keywords: Contact, friction, augmented Lagrangian, generalized Newton's method, Nitsche's method.

\section{Introduction}

The numerical resolution of elastostatic or elastodynamic contact problems is already the subject of an important literature. The algorithms proposed to solve the approximated problem are quite varied. It includes interior points methods $[32,38]$, specific methods for linear complementarity problems such as the Lemke algorithm [27, 8], projected conjugate gradient [35], SSOR with projection $[8,25]$ and finally generalized Newton's methods. This paper focuses on generalized Newton's methods which are widely used, especially in commercial software, and proved their efficiency and robustness in the framework of large scale elasticity problems with contact and friction.

The pioneering work in this area is the one of P. Alart and A. Curnier [2, 3]. The Newton's method is applied to the optimality system of an augmented Lagrangian whose saddle point is the solution to the contact problem with Tresca friction. The optimality system can be easily extended to Coulomb friction. The term "generalized Newton's method" comes from the fact that the optimality system is not continuously differentiable but only Lipschitz-continuous and piecewise continuously differentiable. However, no special treatment is really needed by this nondifferentiable character mainly because from a numerical viewpoint, it is quite improbable to come across a non-differentiable point. Note that in these references, the augmented Lagrangian is written on a nodal discretization of the contact problem, not on the continuous problem.

A second important work is the one of T.A. Laursen and J.C. Simo [40, 31]. It is based on the optimality system of the same augmented Lagrangian. The main difference is that the proposed 
algorithm is an Uzawa algorithm on the contact stress, with a descent parameter equal to the augmentation parameter of the Lagrangian. The generalized Newton's method is only applied on the optimization step of the Uzawa algorithm which only concerns the displacement. This method is widely used, especially in commercial software. This might be due to the fact that it does not require a coupled resolution of the displacement and contact stress. Another characteristic is the fact that the first iteration, if the initial contact stress is set to zero, corresponds to the solving of a penalized contact problem with a penalization coefficient equal to the augmentation parameter of the Lagrangian. It allows to optionally stop after the first iteration if a penalization approach is chosen or to perform the Uzawa iterations to converge toward the non-penalized problem. Moreover, when the augmentation parameter is large enough, the Uzawa algorithm is converging in a few iterations.

P. Alart in [1] gives a global convergence result for a generalized Newton's method applied on a simplified non-symmetric version of the optimality system of the augmented Lagrangian. This global convergence occurs only on a model problem (membrane with obstacle) without the addition of any line search strategy. He also gives and analyzes some examples of instabilities for the case of a two-dimensional elasticity problem with contact on the boundary.

P.W. Christensen and J.S. Pang in [11] prove that the optimality system of the problem approximated by finite elements and extended to Coulomb friction is semi-smooth. A Lipschitzcontinuous map $f$ is said to be semi-smooth at a point $y$ when it satisfies $\sup _{M \in \partial f(y+s)} \| f(y+$ $s)-f(y)-M s \|=o(\|s\|)$ where $\partial f$ is its generalized gradient. The Newton method applied to a semi-smooth map converges super-linearly for a starting point sufficiently close to the solution (see $[34,42])$.

S. Hber and B. Wohlmuth in [22] present an active set strategy. The set of active constraints is updated at each iteration. This algorithm can be re-interpreted as a generalized Newton's method for Alart-Curnier augmented Lagrangian.

K. Kunish and G. Stadler in [41, 29] state that the optimality system of Alart-Curnier augmented Lagrangian is not semi-smooth in the continuous framework. They deduce that the scalability of Newton's method cannot be guarantied. They also prove that the Uzawa algorithm introduced by Simo and Laursen converges whatever the value of the augmentation parameter for the contact with Tresca friction (in a continuous framework, which prove its scalability) and with a number of iterations which decreases when the augmentation parameter increases.

Recently, several attempts have been made to adapt Nitche's method, initially proposed to prescribe a Dirichlet boundary condition, to contact with or without friction (see $[18,43,9]$ ). The advantage of Nitsche-based methods is to prevent the use of a multiplier while being consistent.

In this paper, some generalized Newton's methods based on new formulations of the contact problem are presented. These formulations do not derive from some augmented Lagrangians. The goal of the paper is to compare these new strategies to the existing ones from a numerical experiment viewpoint, especially to test the sensitivity to the augmentation parameter, to the applied load and the efficiency to solve two and three-dimensional problems.

The outline of the paper is the following. The so-called Signorini with Coulomb friction problem is recalled in Section 2, in strong and weak formulations. In Section 3, two versions of the finite element discretization are presented: one with a nodal discretization of the contact condition and another one with an integral one. Additionaly, the numerical tests performed in the next sections are described. Section 4 is devoted to Alart-Curnier generalized Newton's method, Section 5 to the Simo-Laursen method, Section 6 to some new Nitsche-based methods for contact with friction and Section 7 to some other new methods based on augmented multipliers. Finally some conclusions are given in Section 8. 


\section{Signorini's problem with Coulomb friction}

For the sake of simplicity, we consider the classical simple situation of the equilibrium of a linearly elastic body in contact with (static) Coulomb friction with a rigid foundation. Note that the main interest of this problem is to be similar to the one obtained by applying a time integration scheme on a quasi-static problem.

Let $\Omega \subset \mathbb{R}^{d}, d \in\{2,3\}$ be a bounded regular domain which represents the reference configuration of a linearly elastic body submitted to a Neumann condition on $\Gamma_{N}$, a Dirichlet condition on $\Gamma_{D}$ and a unilateral contact condition with friction on $\Gamma_{C}$ with a rigid foundation, where $\Gamma_{N}$, $\Gamma_{D}$ and $\Gamma_{C}$ are non-overlapping open parts of $\partial \Omega$, the boundary of $\Omega$ (see Fig. 1). The part $\Gamma_{D}$ is supposed of non-zero measure in $\partial \Omega$.

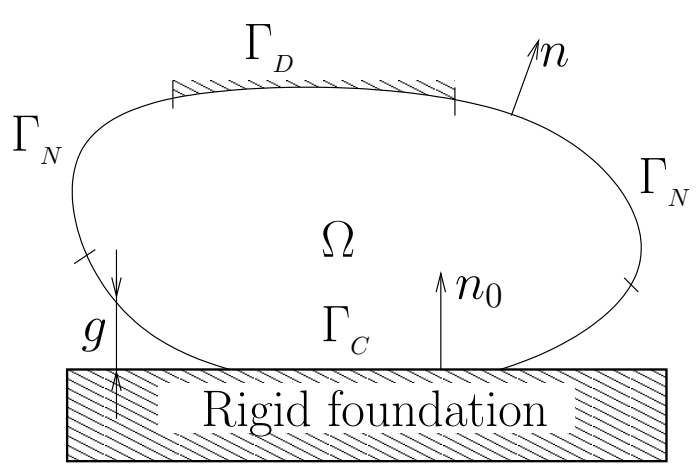

Figure 1: Linearly elastic body $\Omega$ in contact with a rigid foundation.

The displacement $u(t, x)$ of the body obeys the following equations:

$-\operatorname{div} \sigma(u)=f, \quad$ in $\Omega, \quad \sigma(u)=\mathscr{A} \varepsilon(u), \quad$ in $\Omega, \quad \sigma(u) n=k, \quad$ on $\Gamma_{N}, \quad u=0, \quad$ on $\Gamma_{D}$,

where $\sigma(u)$ is the stress tensor, $\varepsilon(u)=\left(\nabla u+\nabla u^{T}\right) / 2$ is the linearized strain tensor, $\mathscr{A}$ is the fourth order elasticity tensor which satisfies usual conditions of symmetry, coercivity and boundedness, $n$ is the outward unit normal to $\Omega$ on $\partial \Omega$ and $f, k$ are given force densities. On $\Gamma_{C}$, it is usual to decompose the displacement and the stress in normal and tangential components as follows, assuming the shape of the rigid foundation to have the $\mathscr{C}^{1}$ regularity:

$$
u_{N}=-u \cdot n_{0}, \quad u_{T}=u+u_{N} n_{0}, \quad \sigma_{N}(u)=-(\sigma(u) n) \cdot n_{0}, \quad \sigma_{T}(u)=\sigma(u) n+\sigma_{N}(u) n_{0},
$$

where $n_{0}$ is the unit outward normal to the obstacle (see Fig. 1). Denoting by $g$ the initial normal gap between the solid and the rigid obstacle, the unilateral contact condition is expressed by the following complementary condition:

$$
u_{N}-g \leq 0, \sigma_{N}(u) \leq 0,\left(u_{N}-g\right) \sigma_{N}(u)=0,
$$

while the static Coulomb friction condition is expressed as follows for $\mathscr{F}$ the friction coefficient:

$$
\left|\sigma_{T}\right| \leq-\mathscr{F} \sigma_{N}, \quad \text { if } u_{T} \neq 0 \text { then } \sigma_{T}=-\mathscr{F} \sigma_{N} \frac{u_{T}}{\left|u_{T}\right|} .
$$

A classical weak formulation (see [14]) can be obtained introducing

$$
V=\left\{v \in H^{1}\left(\Omega ; \mathbb{R}^{d}\right): v=0 \text { on } \Gamma_{D}\right\}, \quad K=\left\{v \in V: v_{N}-g \leq 0 \text { on } \Gamma_{C}\right\},
$$




$$
\begin{gathered}
a(u, v)=\int_{\Omega} \mathscr{A} \varepsilon(u): \varepsilon(v) d x, \quad l(v)=\int_{\Omega} f . v d x+\int_{\Gamma_{N}} k . v d \Gamma . \\
W_{N}=\left\{v \in L^{2}\left(\Gamma_{C}\right): \exists w \in V, v=w_{N}\right\}, \quad W_{T}=\left\{v \in L^{2}\left(\Gamma_{C} ; \mathbb{R}^{d-1}\right): \exists w \in V, v=w_{T}\right\}, \\
W=W_{N} \times W_{T}, \quad j(s, v)=\left\langle s,\left|v_{T}\right|\right\rangle_{W_{N}^{\prime}, W_{N}} .
\end{gathered}
$$

The space $W_{N}$ (resp. $W_{T}$ ) is the space of the normal (resp. tangential) traces and $W_{N}^{\prime}$ (resp. $W_{T}^{\prime}$ ) will denote its topological dual space. Note that the duality pairing $\langle\cdot, \cdot\rangle_{W_{N}^{\prime}, W_{N}}$ reduces to the integral on $\Gamma_{C}$ when the contact stress $\sigma_{N}(u)$ is sufficiently regular. Problem (1) - (3) is then formally equivalent to the variational inequality (see [14])

$$
\left\{\begin{array}{l}
\text { Find } u \in K \text { satisfying } \\
a(u, v-u)+j\left(-\mathscr{F} \sigma_{N}(u), u\right)-j\left(-\mathscr{F} \sigma_{N}(u), v\right) \geq l(v-u), \quad \forall v \in K .
\end{array}\right.
$$

A generalized Newton's method cannot be directly applied to this formulation. Classically, one introduces some multipliers representing the contact and friction stresses and obtains the following hybrid formulation (see [4] for instance):

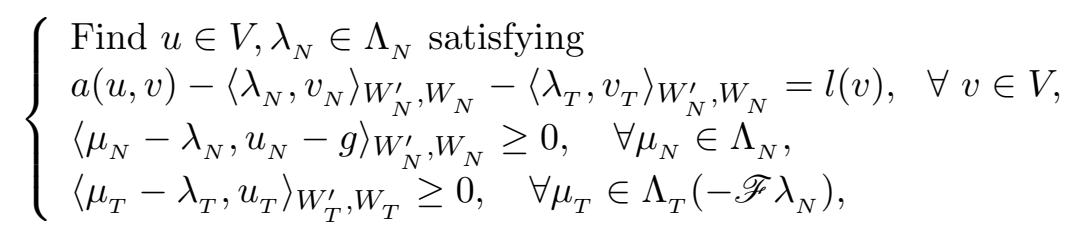

with $\Lambda_{N}$ the cone of non-positive contact stresses

$$
\Lambda_{N}=\left\{\lambda_{N} \in W_{N}^{\prime}:\left\langle\lambda_{N}, v_{N}\right\rangle_{W_{N}^{\prime}, W_{N}} \geq 0 \quad \forall v \in K\right\}
$$

and $\Lambda_{T}(s)$ the set of admissible friction stresses defined by

$$
\Lambda_{T}(s)=\left\{\lambda_{T} \in W_{T}^{\prime}:-\left\langle\lambda_{T}, v_{T}\right\rangle_{W_{T}^{\prime}, W_{T}}+\left\langle s,\left|v_{T}\right|\right\rangle_{W_{N}^{\prime}, W_{N}} \geq 0 \quad \forall v_{T} \in W_{T}\right\} .
$$

In the case of Tresca friction, the (weakly) non-negative threshold $s \in W_{N}^{\prime}$ is given and one considers the problem

$$
\left\{\begin{array}{l}
\text { Find } u \in V, \lambda_{N} \in \Lambda_{N} \text { satisfying } \\
a(u, v)-\left\langle\lambda_{N}, v_{N}\right\rangle_{W_{N}^{\prime}, W_{N}}-\left\langle\lambda_{T}, v_{T}\right\rangle_{W_{N}^{\prime}, W_{N}}=l(v), \quad \forall v \in V \\
\left\langle\mu_{N}-\lambda_{N}, u_{N}-g\right\rangle_{W_{N}^{\prime}, W_{N}} \geq 0, \quad \forall \mu_{N} \in \Lambda_{N}, \\
\left\langle\mu_{T}-\lambda_{T}, u_{T}\right\rangle_{W_{T}^{\prime}, W_{T}} \geq 0, \quad \forall \mu_{T} \in \Lambda_{T}(s),
\end{array}\right.
$$

The formulation (6) is the optimality system of the Lagrangian

$$
\mathscr{L}(u, \lambda)=\frac{1}{2} a(u, u)-l(u)-j(s, u)-\left\langle\lambda, u_{N}-g\right\rangle_{W^{\prime}, W}-I_{\Lambda_{N}}\left(\lambda_{N}\right)-I_{\Lambda_{T}(s)}\left(\lambda_{T}\right),
$$

where $I_{\Lambda_{N}}$ (resp. $\left.I_{\Lambda_{T}(s)}\right)$ is the indicator function of $\Lambda_{N}$ (resp. $\Lambda_{T}(s)$ ) (see for instance [30] for more details). This last term means that the saddle point has to be chosen with the constraints $\lambda_{N} \in \Lambda_{N}$ and $\lambda_{T} \in \Lambda_{T}(s)$. The fact that the Lagrangian $\mathscr{L}(u, \lambda)$ is under constraint poses practical difficulties for the numerical resolution. This is one of the reason why augmented Lagrangian are considered. 
The existence and uniqueness of the solution to Problem (6) is obtained by standard techniques (see [26] for instance). Unfortunately, as it is well known, the problem with Coulomb friction (5) is not a variational problem and thus do not derive from a Lagrangian. Some existence results for the problem with Coulomb friction have been proved in [23] and [15] with some reasonable assumptions on the regularity of the boundary and for a sufficiently small friction coefficient. The uniqueness of the solution for a small friction coefficient is an open problem. A criterion has been given in [36] which states that the uniqueness is reached under a kind of regularity condition on the solution and for a small enough friction coefficient. Examples of non-uniqueness has been exhibited in $[19,20]$ for a large friction coefficient.

\section{$3 \quad$ Finite element approximation}

In the framework of the finite element method, different principles can be used to approximate a contact condition with or without friction (see $[26,25]$ for instance). The contact condition can be applied on each finite element node of the contact boundary (or alternatively on the Gauss points of a quadrature method defined on the contact boundary). In that case, generally, the non-interpenetration condition is prescribed on each node. The contact condition can also be weakened by the use of a multiplier of lower degree and a contact condition on each finite element node of the multiplier. In that case, this is generally the non-positivity on each node of the multiplier which is considered. A third possibility, used more or less implicitly is some references (for instance in $[30,39]$ ) is to use directly a non constrained integral weak formulation of the contact condition. From the resolution with a Newton algorithm point of view, the two first alternatives are very close. Thus, the choice is made to test only the first and the third alternatives. In the following, we will denote by $V^{h}$ a family of finite dimensional vector spaces (see [7]) indexed by $h$ coming from a family $\mathscr{T}^{h}$ of meshes of the domain $\Omega\left(h=\max _{T \in \mathcal{T}^{h}} h_{T}\right.$ where $h_{T}$ is the diameter of $T$ ).

\subsection{Nodal discretization of the contact condition}

This is probably the most commonly used method to approximate the contact condition. It consists to prescribe the non-penetration and friction conditions on each finite element node of a Lagrange finite element method. This a priori restricts $V^{h}$ to be built from a Lagrange finite element method. This means that denoting $\phi_{i}(x), i=1 . . N_{d}$ the (scalar) shapes functions of $V^{h}$ and $a_{i} \in \mathbb{R}^{d}$ the corresponding finite element nodes, one has $\phi_{i}\left(a_{j}\right)=\delta_{i j}$ and the following Lagrange interpolation principle:

$$
u^{h}(x)=\sum_{i=1}^{N_{d}} u^{h}\left(a_{i}\right) \varphi_{i}(x) .
$$

Let us denote $\mathcal{N}_{\Gamma_{C}}$ the set of node indices on the contact boundary. The approximation of Problem (5) with a nodal contact condition reads as:

$$
\left\{\begin{array}{l}
\text { Find } u^{h} \in V^{h}, \lambda_{N}^{i} \in \mathbb{R}, \lambda_{T}^{i} \in \mathbb{R}^{d-1}, i \in \mathcal{N}_{\Gamma_{C}} \text {, satisfying } \\
a\left(u^{h}, v^{h}\right)-\sum_{i \in \mathcal{N}_{\Gamma_{C}}} \lambda_{N}^{i} v_{N}^{h}\left(a_{i}\right)-\sum_{i \in \mathcal{N}_{\Gamma_{C}}} \lambda_{T}^{i} \cdot v_{T}^{h}\left(a_{i}\right)=l\left(v^{h}\right), \quad \forall v \in V^{h}, \\
\lambda_{N}^{i} \leq 0, \quad u_{N}^{h}\left(a_{i}\right)-g\left(a_{i}\right) \leq 0, \quad \lambda_{N}^{i}\left(u_{N}^{h}\left(a_{i}\right)-g\left(a_{i}\right)\right)=0 \quad \forall i \in \mathcal{N}_{\Gamma_{C}}, \\
\left|\lambda_{T}^{i}\right| \leq-\mathscr{F} \lambda_{N}^{i}, \quad \text { if } u_{T}^{h}\left(a_{i}\right) \neq 0 \text { then } \lambda_{T}^{i}=\mathscr{F} \lambda_{N}^{i} \frac{u_{T}^{h}\left(a_{i}\right)}{\left|u_{T}^{h}\left(a_{i}\right)\right|} \quad \forall i \in \mathcal{N}_{\Gamma_{C}} .
\end{array}\right.
$$


An algebraic formulation can be obtained by defining the stiffness matrix $K$ and the righthand side $L$ as

$$
K_{(i-1) d+j,(k-1) d+l}=a\left(\varphi_{i} e_{j}, \varphi_{k} e_{l}\right), \quad L_{(i-1) d+j}=\ell\left(\varphi_{i} e_{j}\right), \quad 1 \leq i, k \leq N_{d}, \quad 1 \leq j, l \leq d,
$$

where $e_{0}, \cdots, e_{d-1}$ is the canonical basis of $\mathbb{R}^{d}$. Let also $U$ be the vector of degrees of freedom such that

$$
U_{(i-1) d+j}=u^{h}\left(a_{i}\right) \cdot e_{j},
$$

and $N_{i} \in \mathbb{R}^{d N_{d}}, T_{i} \in \mathcal{M}_{d N_{d},(d-1)}(\mathbb{R})$ for $i \in \mathcal{N}_{\Gamma_{C}}$ be defined such that

$$
N_{i}^{T} U=u_{N}^{h}\left(a_{i}\right), \quad T_{i}^{T} U=u_{T}^{h}\left(a_{i}\right) .
$$

Let also $B_{N}$ be the matrix whose lines are the vectors $N_{i}, B_{T}$ be the matrix whose lines are the columns of $T_{i}, \Lambda_{N}$ the vector of all $\lambda_{N}^{i}$ and $\Lambda_{T}$ the vector of all $\lambda_{T}^{i}$. Then the algebraic formulation of Problem (8) read as

$$
\left\{\begin{array}{l}
\text { Find } U \in \mathbb{R}^{d N_{d}}, \lambda_{N}^{i} \in \mathbb{R}, \lambda_{T}^{i} \in \mathbb{R}^{d-1}, i \in \mathcal{N}_{\Gamma_{C}}, \text { satisfying } \\
K U-B_{N}^{T} \Lambda_{N}-B_{T}^{T} \Lambda_{T}=L, \\
\lambda_{N}^{i} \leq 0, \quad N_{i}^{T} U-g\left(a_{i}\right) \leq 0, \quad \lambda_{N}^{i}\left(N_{i}^{T} U-g\left(a_{i}\right)\right)=0, \quad \forall i \in \mathcal{N}_{\Gamma_{C}}, \\
\left|\lambda_{T}^{i}\right| \leq-\mathscr{F} \lambda_{N}^{i}, \quad \text { if } T_{i}^{T} U \neq 0 \text { then } \lambda_{T}^{i}=\mathscr{F} \lambda_{N}^{i} \frac{T_{i}^{T} U}{\left|T_{i}^{T} U\right|} \quad \forall i \in \mathcal{N}_{\Gamma_{C}} .
\end{array}\right.
$$

This is a classical result that Problem (8) admits a solution whatever the value of the friction coefficient $\mathscr{F} \geq 0$ and that this solution is unique for $\mathscr{F}$ small enough of order $O(\sqrt{h})$ (see [17]).

It is also well known that in the two-dimensional case, Problem (9) is equivalent to a linear complementarity problem (LCP, see [12]). The three dimensional frictional problem cannot be expressed as a linear complementarity problem without a polyhedral approximation of the Coulomb friction cone [28]. However, it can be expressed as a second order cone linear complementarity problem (SOCLCP, see [24]).

\subsection{Integral approximation of the contact condition}

In that case, $V^{h}$ is an arbitrary finite element space and, additionally, we denote by $W_{N}^{h} \subset L^{2}\left(\Gamma_{D}\right)$, $W_{T}^{h} \subset L^{2}\left(\Gamma_{D} ; \mathbb{R}^{d-1}\right), W^{h}=W_{N}^{h} \times W_{T}^{h}$ some finite element spaces for the approximation of contact forces. We use the following equivalent expression of contact and friction condition (other choices are presented in the next sections):

$$
\begin{aligned}
\lambda_{N}^{h} & =-\left(\lambda_{N}^{h}-r\left(u_{N}^{h}-g\right)\right)_{-}, \\
\lambda_{T}^{h} & =P_{B\left(0, \mathscr{F}\left(\lambda_{N}^{h}-r\left(u_{N}^{h}-g\right)\right)_{-}\right)}\left(\lambda_{T}^{h}-r u_{T}^{h}\right),
\end{aligned}
$$

where $r>0$ is an augmentation parameter, $P_{B}(0, \rho)$ is the orthogonal projection on the closed ball of center 0 and radius $\rho$ and $(\cdot)_{-}$is the negative part $\left((x)_{-}=0\right.$ for $x \geq 0$ and $(x)_{-}=-x$ for $x \leq 0)$. In particular, these expressions can be derived from Alart-Curnier Augmented Lagrangian which will be described later on. 
This allows to give the following approximation of Problem (5) with an unconstrained integral contact condition:

$$
\left\{\begin{array}{l}
\text { Find } u^{h} \in V^{h}, \lambda_{N}^{h} \in W_{N}^{h} \text { and } \lambda_{T}^{h} \in W_{T}^{h} \text { satisfying } \\
a\left(u^{h}, v^{h}\right)-\int_{\Gamma_{C}} \lambda_{N}^{h} v_{N}^{h} d \Gamma-\int_{\Gamma_{C}} \lambda_{T}^{h} \cdot v_{T}^{h} d \Gamma=l\left(v^{h}\right), \quad \forall v \in V^{h}, \\
-\frac{1}{r} \int_{\Gamma_{C}}\left(\lambda_{N}^{h}+\left(\lambda_{N}^{h}-r\left(u_{N}^{h}-g\right)\right)_{-}\right) \mu_{N}^{h} d \Gamma \\
\quad-\frac{1}{r} \int_{\Gamma_{C}}\left(\lambda_{T}^{h}-P_{B\left(0, \mathscr{F}\left(\lambda_{N}^{h}-r\left(u_{N}^{h}-g\right)\right)_{-}\right)}\left(\lambda_{T}^{h}-r u_{T}^{h}\right)\right) \cdot \mu_{T}^{h} d \Gamma=0 \quad \forall \mu^{h} \in W^{h} .
\end{array}\right.
$$

The existence and uniqueness of a solution to Problem (12) is discussed in [30]. The inf-sup (or LBB) condition

$$
\inf _{\mu^{h} \in W^{h}} \sup _{v^{h} \in V^{h}} \frac{\int_{\Gamma_{C}} \mu^{h} \cdot v^{h} d \Gamma}{\left\|v^{h}\right\|_{V}\left\|\mu^{h}\right\|_{W}} \geq \gamma>0,
$$

is necessary to ensure the uniqueness of the multiplier. A constant $\gamma>0$ independent of $h$ is necessary to obtain an optimal numerical convergence. Note that, unlike Problem (8), Problem (12) is not equivalent to a LCP or a SOCLCP problem.

\subsection{Description of the numerical experiments}

The aim of the numerical experiments presented in the next sections is to compare the efficiency of the different generalized Newton's method on a small set of simple contact situations in two and three dimensions. Three different two-dimensional situations are considered: an elastic punch clamped on its top submitted to two different prescribed vertical compressions and in contact with a flat rigid obstacle at the bottom (see the mesh in Fig. 2 and the two deformations in Fig. $3)$ and a disk with no Dirichlet condition $\left(\Gamma_{D}=\emptyset\right)$ submitted to a vertical volumic load and also in contact with a flat rigid obstacle at the bottom (mesh and deformation are also in Fig. 2 and Fig. 3, respectively). Both are in plane stress approximation. The deformations, especially for the second case, are obviously non-physical since they are too important for the linear elasticity model to remain valid. This is done intentionally here to test the algorithms both with a small and a large real contact area and to test the influence of the size of the load.

In 3D, we consider the counterpart situations for a 3D punch and a sphere (see Fig. 4 and Fig. 5 for the meshes and the deformations, respectively).

For all the experiment a quadratic isoparametric finite element is considered. The number of displacement degrees of freedom for the different meshes used in the numerical experiments are summarized in Table 1.

\begin{tabular}{|c|c|c|c|c|c|c|c|}
\hline \multicolumn{2}{|c|}{ 2D punch } & \multicolumn{2}{c|}{ Disc } & \multicolumn{2}{c|}{ 3D punch } & \multicolumn{2}{c|}{ Sphere } \\
\hline$h=4$ & 424 & $h=8$ & 150 & $h=5$ & 1182 & $h=20$ & 75 \\
\hline$h=1$ & 6226 & $h=2$ & 2782 & $h=3$ & 4305 & $h=6$ & 2232 \\
\hline$h=0.25$ & 97390 & $h=0.5$ & 45706 & $h=1.7$ & 24375 & $h=2.3$ & 26259 \\
\hline
\end{tabular}

Table 1: Number of displacement degrees of freedom for the different meshes used.

Note that the two cases corresponding to the disc and the sphere are semi-coercive cases in the sense that the bilinear form $a(\cdot, \cdot)$ is not strictly coercive due to free rigid body motions. Nevertheless, there exists a unique solution because the load is chosen compatible. 
The different parameters used for the model are a Poisson ratio $\nu=0.25$, a Young modulus $E=2660 M P a$ in $2 \mathrm{D}$ cases and $E=2500 M P a$ in $3 \mathrm{D}$ cases and a uniform (excessive) volumic load of $20 \times 10^{6} \mathrm{~N} / \mathrm{m}^{3}$ for the disc and the sphere.

The initial iteration for the different (generalized Newton or Uzawa) algorithms is the reference configuration (zero displacement). A very basic line search is considered for generalized Newton's method which can be summarized as follows :

- Test a full Newton step. Since the norm of the residual is greater than the one of the previous iteration, divide the step by a factor 2 , with a minimal step of $10^{-10}$.

- If there is more than three Newton iterations with a decreasing of the residual less than $1 \%$ then allow a step with an increase of the residual of a factor 2 .
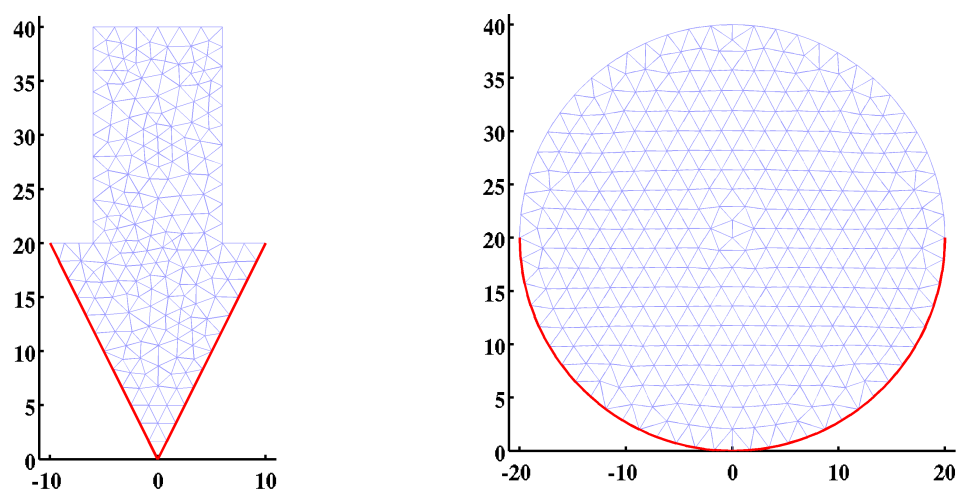

Figure 2: 2D meshes.
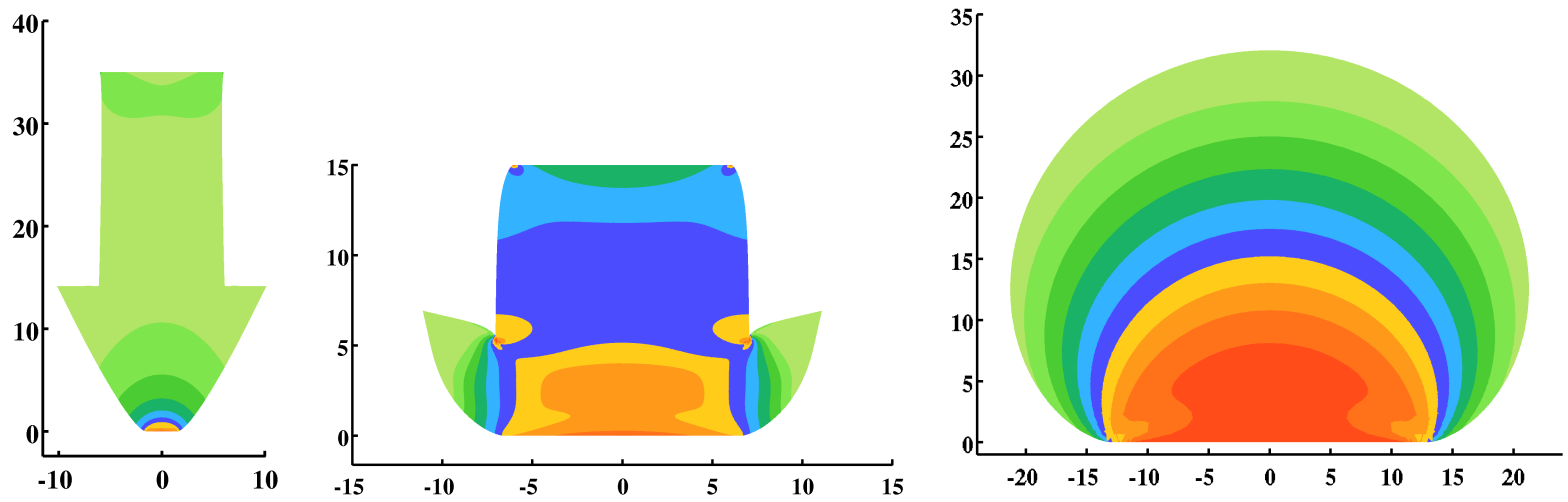

Figure 3: 2D deformed configurations with color plot of the Von Mises stress (and with friction, $\mathscr{F}=1)$.

Let us recall that generalized Newton's method means that Newton's method is applied on a discrete system like (12) which is not $\mathscr{C}^{1}$-regular but only Lipschitz-continuous and piecewise $\mathscr{C}^{1}$-regular. No special treatment is applied when a point of non-differentiability is reached by Newton's method. Such a point is on the frontier between two or more zones of differentiability and the tangent matrix corresponding to one of these zones is selected arbitrarily. 
In the sections that follow, the graphs represent the number of iterations of Newton's method to achieve convergence. Each of the six graphs of a set corresponds to one of the six situations described previously. The number of iterations is limited to 100. A number of iteration of 100 means that the algorithm has been stopped.
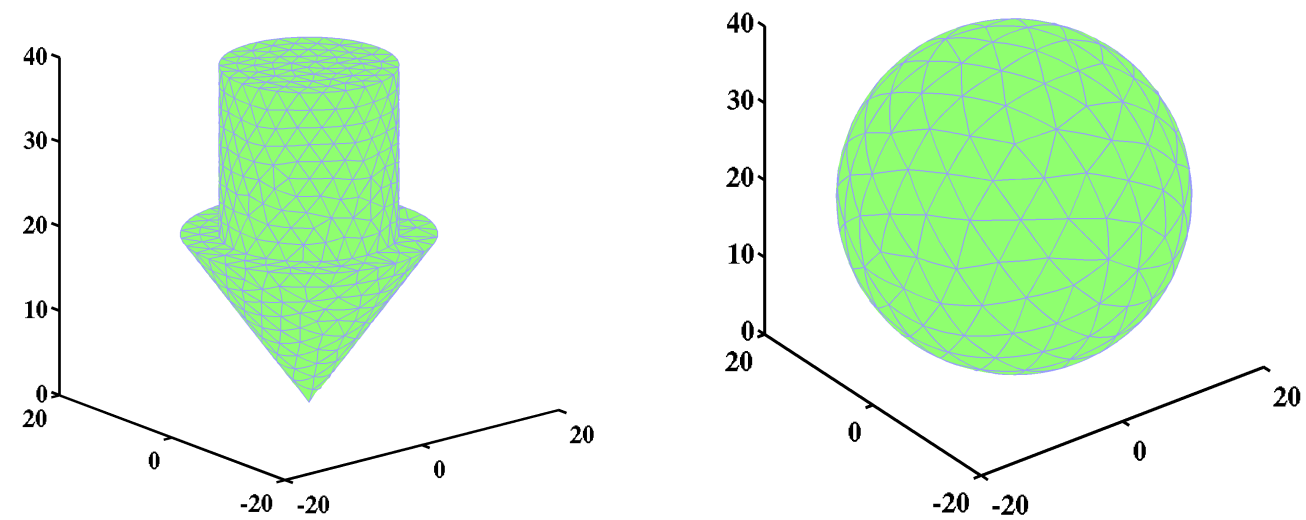

Figure 4: 3D meshes.
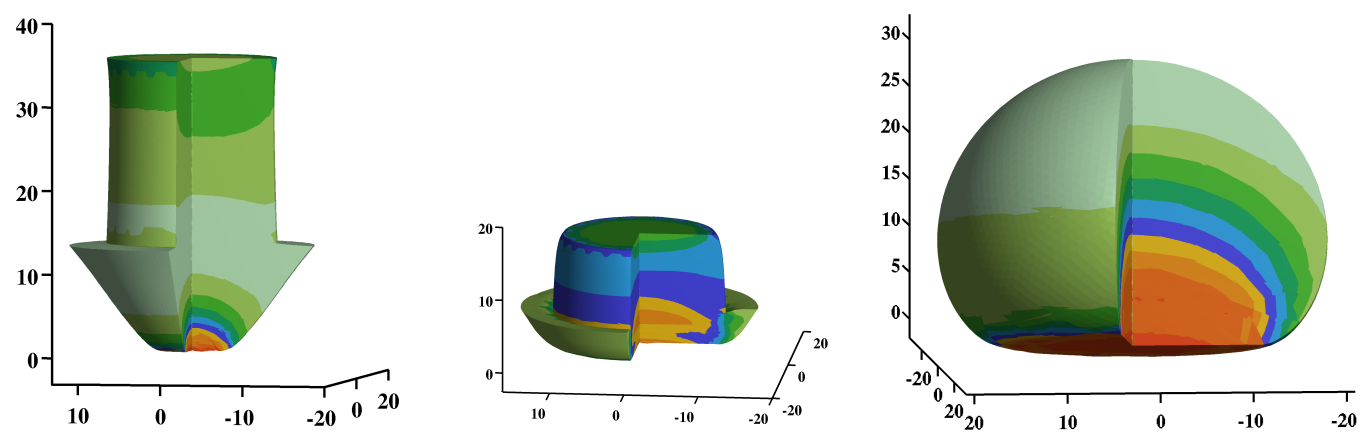

Figure 5: 3D deformed configurations with color plot of the Von Mises stress (and with friction, $\mathscr{F}=1)$.

\section{Alart-Curnier generalized Newton's method}

Most of generalized Newton's methods used to solve contact problem in elasticity are based on the augmented Lagrangian formulation presented in $[2,3]$ on the finite element approximation of the contact problem with a nodal contact with friction condition. This formulation can be interpreted as a proximal Lagrangian in the sense of R.T. Rockafellar for the problem with Tresca friction (see $[30,37]$ for more details). In this section, the presentation is made for a contact condition with Coulomb friction for both a nodal and an integral approximation.

First, let us recall that an augmented Lagrangian formulation is only possible for the frictionless case or for the case with Tresca friction since the Coulomb friction law do not derive from a potential. However, the optimality system of the augmented Lagrangian is classically extended to the Coulomb friction law.

In the continuous framework, the augmented Lagrangian has the following expression for the 
problem with Tresca friction:

$$
\begin{aligned}
\mathscr{L}_{r}(u, \lambda) & =\frac{1}{2} a(u, u)-\ell(u)-\frac{1}{2 r} \int_{\Gamma_{C}}|\lambda|^{2} d \Gamma \\
& +\frac{1}{2 r} \int_{\Gamma_{C}}\left(\lambda_{N}-r\left(u_{N}-g\right)\right)_{-}^{2} d \Gamma+\frac{1}{2 r} \int_{\Gamma_{C}}\left|P_{B(0, s)}\left(\lambda_{T}-r u_{T}\right)\right|^{2} d \Gamma .
\end{aligned}
$$

where $s$ is the Tresca threshold. Note that this expression is only valid for sufficiently regular Tresca threshold and friction stresses in $L^{2}\left(\Gamma_{C}\right)$. The advantage compared to $(7)$ is that there is no constraint on the augmented Lagrangian variables. With $H=L^{2}\left(\Gamma_{C} ; \mathbb{R}^{d}\right)$, the optimality system (still for Tresca friction) read as:

$$
\left\{\begin{array}{l}
a(u, v)=\ell(v)+\int_{\Gamma_{C}}\left(\lambda_{N}-r\left(u_{N}-g\right)\right)_{-} v_{N} d \Gamma+\int_{\Gamma_{C}} P_{B(0, s)}\left(\lambda_{T}-r u_{T}\right) \cdot v_{T} d \Gamma \quad \forall v \in V, \\
-\frac{1}{r} \int_{\Gamma_{C}}\left(\lambda_{N}+\left(\lambda_{N}-r\left(u_{N}-g\right)\right)_{-}\right) \mu_{N} d \Gamma-\frac{1}{r} \int_{\Gamma_{C}}\left(\lambda_{T}-P_{B(0, s)}\left(\lambda_{T}-r u_{T}\right)\right) \cdot \mu_{T} d \Gamma=0 \quad \forall \mu \in H .
\end{array}\right.
$$

This optimality system can be adapted to the Coulomb friction law simply by replacing the threshold $s$ by $-\mathscr{F} \lambda_{N}$. It is in fact more usual to replace it by the so called augmented multiplier $-\mathscr{F}\left(\lambda_{N}-r\left(u_{N}-g\right)\right)_{-}$(see [3]). It is also possible, as for instance in [1], to simplify the first line of the optimality system by using the two other lines and then to obtain the less symmetric following formulation of the problem:

$$
\left\{\begin{array}{l}
a(u, v)=\ell(v)+\int_{\Gamma_{C}} \lambda_{N} v_{N} d \Gamma+\int_{\Gamma_{C}} \lambda_{T} \cdot v_{T} d \Gamma \quad \forall v \in V, \\
-\frac{1}{r} \int_{\Gamma_{C}}\left(\lambda_{N}+\left(\lambda_{N}-r\left(u_{N}-g\right)\right)_{-}\right) \mu_{N} d \Gamma \\
\quad-\frac{1}{r} \int_{\Gamma_{C}}\left(\lambda_{T}-P_{B\left(0, \mathscr{F}\left(\lambda_{N}-r\left(u_{N}-g\right)_{-}\right)\right.}\left(\lambda_{T}-r u_{T}\right)\right) \cdot \mu_{T} d \Gamma=0 \quad \forall \mu \in H .
\end{array}\right.
$$

It is easy to check that this formulation is equivalent to Problem (5) when the solution is assumed to be regular enough $\left(\lambda_{N}\right.$ and $\lambda_{T}$ in $\left.H\right)$.

\subsection{Nodal approximation of the contact condition, unsymmetric version}

The first numerical experiment concerns the approximation of the less symmetric system (14) with a nodal approximation of the contact and friction conditions. The generalized Newton's method is applied on the following system:

$$
\left\{\begin{array}{l}
a\left(u^{h}, v^{h}\right)=\ell\left(v^{h}\right)+\sum_{a \in \mathcal{N}_{\Gamma_{C}}} \lambda_{N}^{a} v_{N}^{h}(a)+\sum_{a \in \mathcal{N}_{\Gamma_{C}}} \lambda_{T}^{a} \cdot v_{T}^{h}(a) \quad \forall v^{h} \in V^{h}, \\
-\frac{1}{r}\left(\lambda_{N}^{a}+\left(\lambda_{N}^{a}-r\left(u_{N}^{h}(a)-g(a)\right)\right)_{-}\right)=0, \quad \forall a \in \mathcal{N}_{\Gamma_{C}}, \\
-\frac{1}{r}\left(\lambda_{T}^{a}-P_{B\left(0, \mathscr{F}\left(\lambda_{N}^{a}-r\left(u_{N}^{h}(a)-g(a)\right)\right)_{-}\right)}\left(\lambda_{T}^{a}-r u_{T}^{h}(a)\right)\right)=0, \quad \forall a \in \mathcal{N}_{\Gamma_{C}} .
\end{array}\right.
$$

The results are shown in Fig. 6 for the frictionless case $(\mathscr{F}=0)$ and in Fig. 7 for the frictional one $(\mathscr{F}=1)$. Concerning the frictionless case (Fig. 6$)$, in the coercive cases (2D and 3D punches) the number of iterations is less than 20 regardless the value of the augmentation parameter $r$, except for one experiment corresponding to the finest mesh of the 3D punch with large deflection. 
Note that there is a slight increase of the number of iterations when the mesh becomes finer which is probably due to the deterioration of the conditioning of the system. Conversely, there is no dependence of the iterations number on the size of the load. Nevertheless, in the non-coercive cases, the method is performing poorly, especially in the case of coarse meshes.
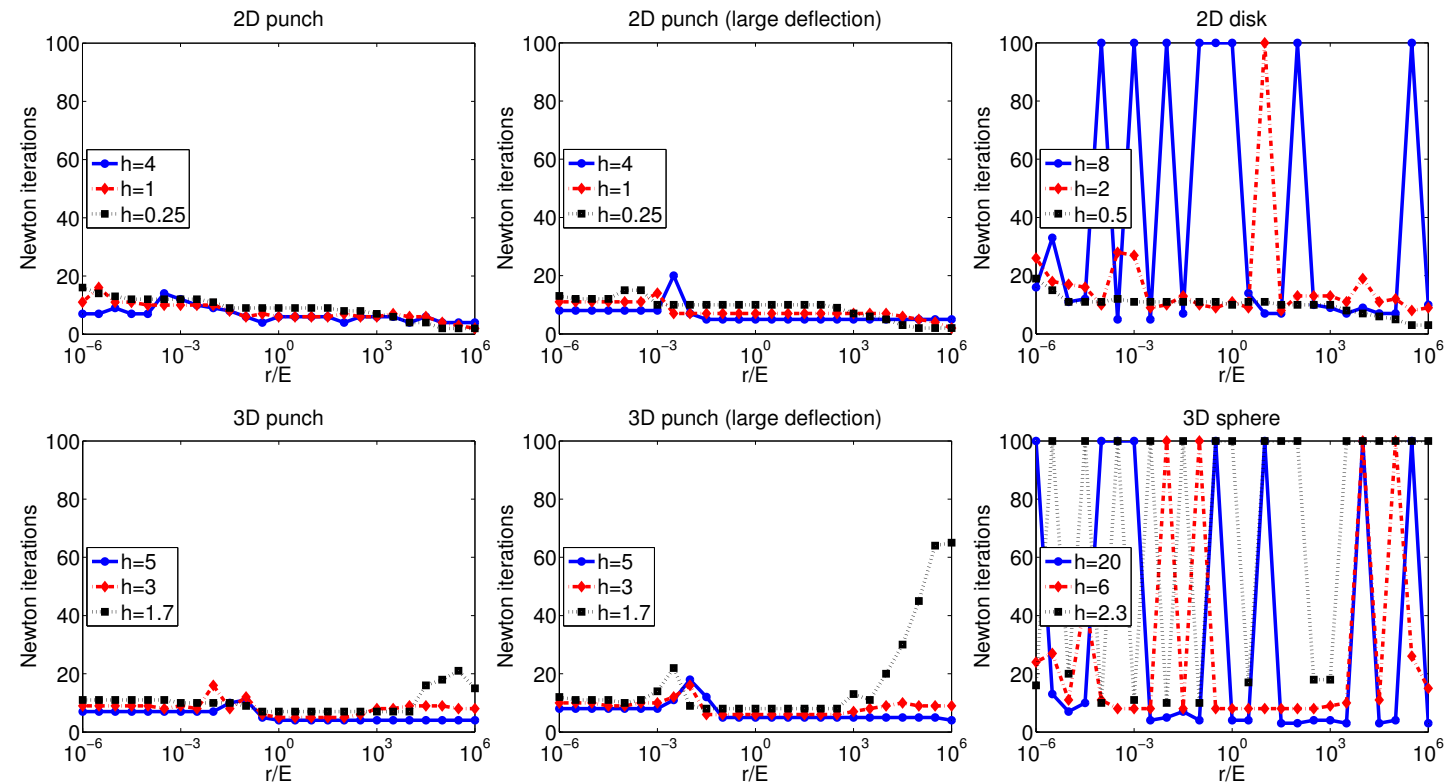

Figure 6: Number of iterations for Alart-Curnier generalized Newton's method with nodal frictionless contact approximation. Unsymmetric version.
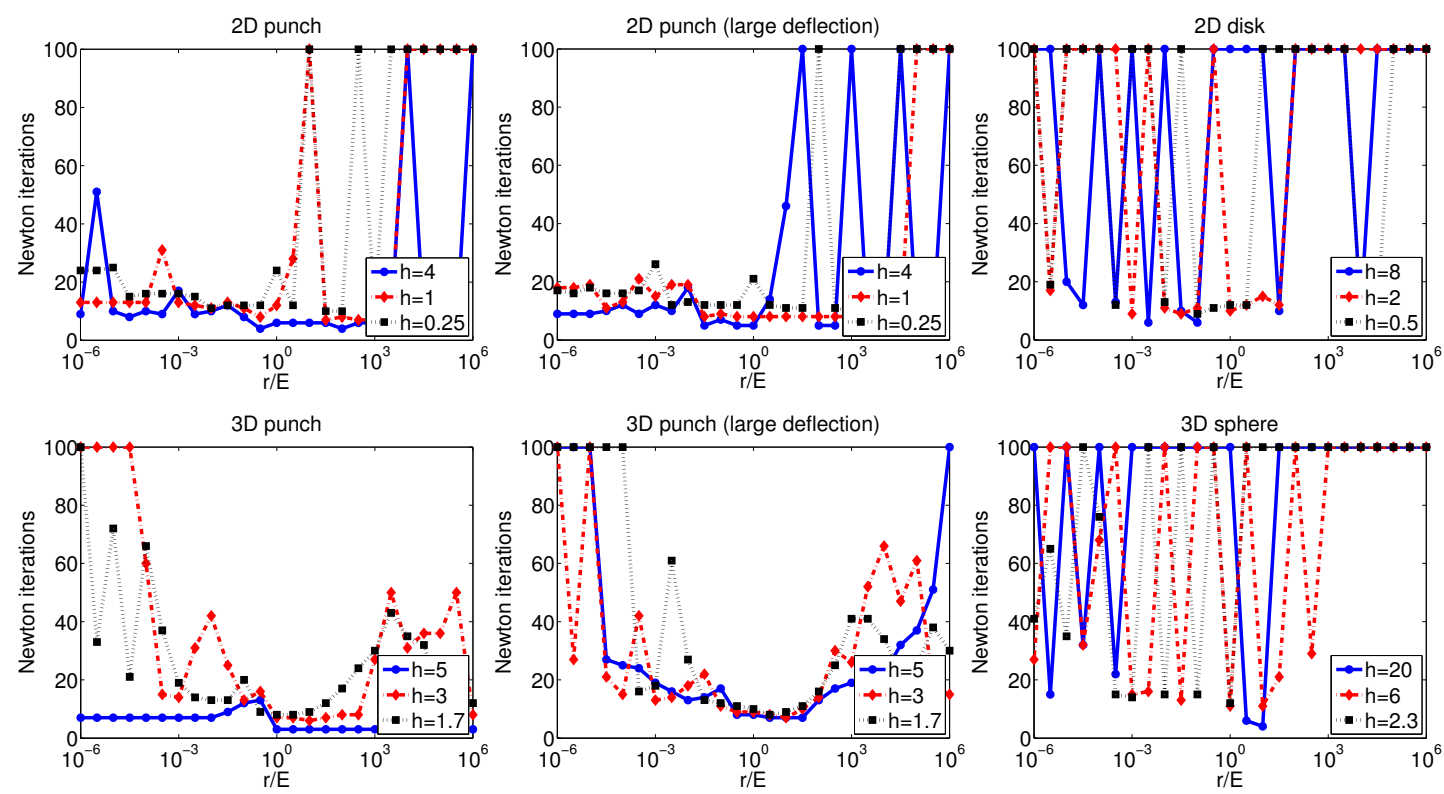

Figure 7: Number of iterations for Alart-Curnier generalized Newton's method with nodal contact with friction approximation. Unsymmetric version. 
Concerning now the coercive cases with friction (Fig. 7), the convergence is less uniform with respect to the augmentation parameter than for the frictionless cases. However, the convergence occurs in less than 20 iterations for a value of the augmentation parameter close to the value of the Young modulus. In the non-coercive cases, the convergence occurs only for a few experiments.

\subsection{Nodal approximation of the contact condition, symmetric version}

In this section, instead of using the less symmetric system, we test the system without the simplification of the first line. This leads to the following formulation of the nodal approximation:

$$
\left\{\begin{aligned}
a\left(u^{h}, v^{h}\right)= & \ell\left(v^{h}\right)+\sum_{a \in \mathcal{N}_{\Gamma_{C}}}\left(\lambda_{N}^{a}-r\left(u_{N}^{h}(a)-g(a)\right)\right)_{-} v_{N}^{h}(a) \\
& +\sum_{a \in \mathcal{N}_{\Gamma_{C}}} P_{B\left(0, \mathscr{F}\left(\lambda_{N}^{a}-r\left(u_{N}^{h}(a)-g(a)\right)\right)_{-}\right)}\left(\lambda_{T}^{a}-r u_{T}^{h}(a)\right) \cdot v_{T}^{h}(a) \quad \forall v^{h} \in V^{h}, \\
-\frac{1}{r}\left(\lambda_{N}^{a}+\right. & \left.\left(\lambda_{N}^{a}-r\left(u_{N}^{h}(a)-g(a)\right)\right)_{-}\right)=0, \quad \forall a \in \mathcal{N}_{\Gamma_{C}}, \\
-\frac{1}{r}\left(\lambda_{T}^{a}-\right. & \left.P_{B\left(0, \mathscr{F}\left(\lambda_{N}^{a}-r\left(u_{N}^{h}(a)-g(a)\right)\right)_{-}\right)}\left(\lambda_{T}^{a}-r u_{T}^{h}(a)\right)\right)=0, \quad \forall a \in \mathcal{N}_{\Gamma_{C}} .
\end{aligned}\right.
$$

The corresponding numerical experiments are presented in Fig. 8 and Fig. 9. There is a clear deterioration of the convergence for the frictionless case (Fig. 8) compared to the less symmetric system except for the non-coercive case. In the frictional case, shown in Fig. 9 the deterioration is less important but is still present especially in 3D. We did not made further investigation to identify the reasons for this deterioration. The fact that there is twice as much projections in the symmetric formulation can be a reason of this deterioration.
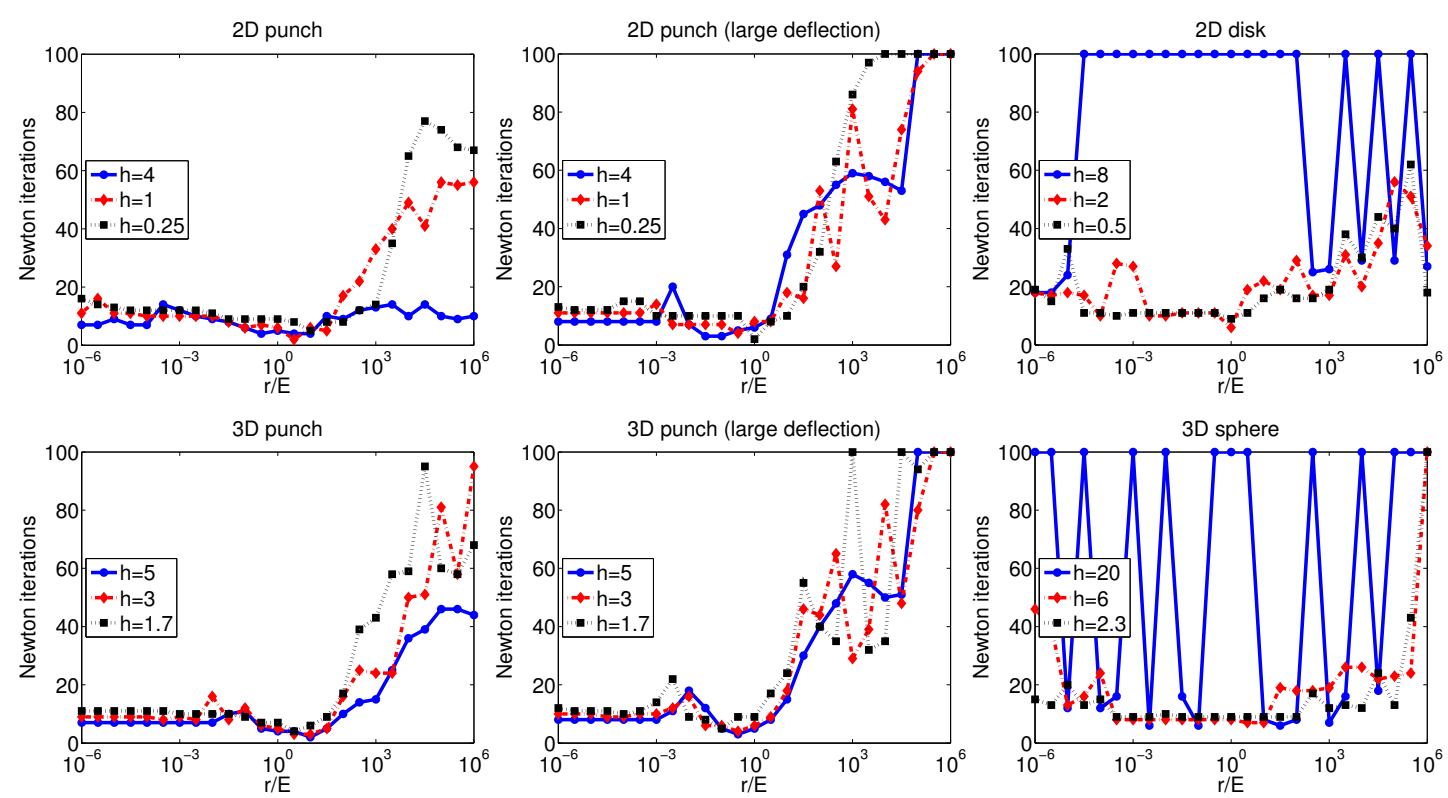

Figure 8: Number of iterations for Alart-Curnier generalized Newton's method with nodal frictionless contact approximation. Symmetric version. 

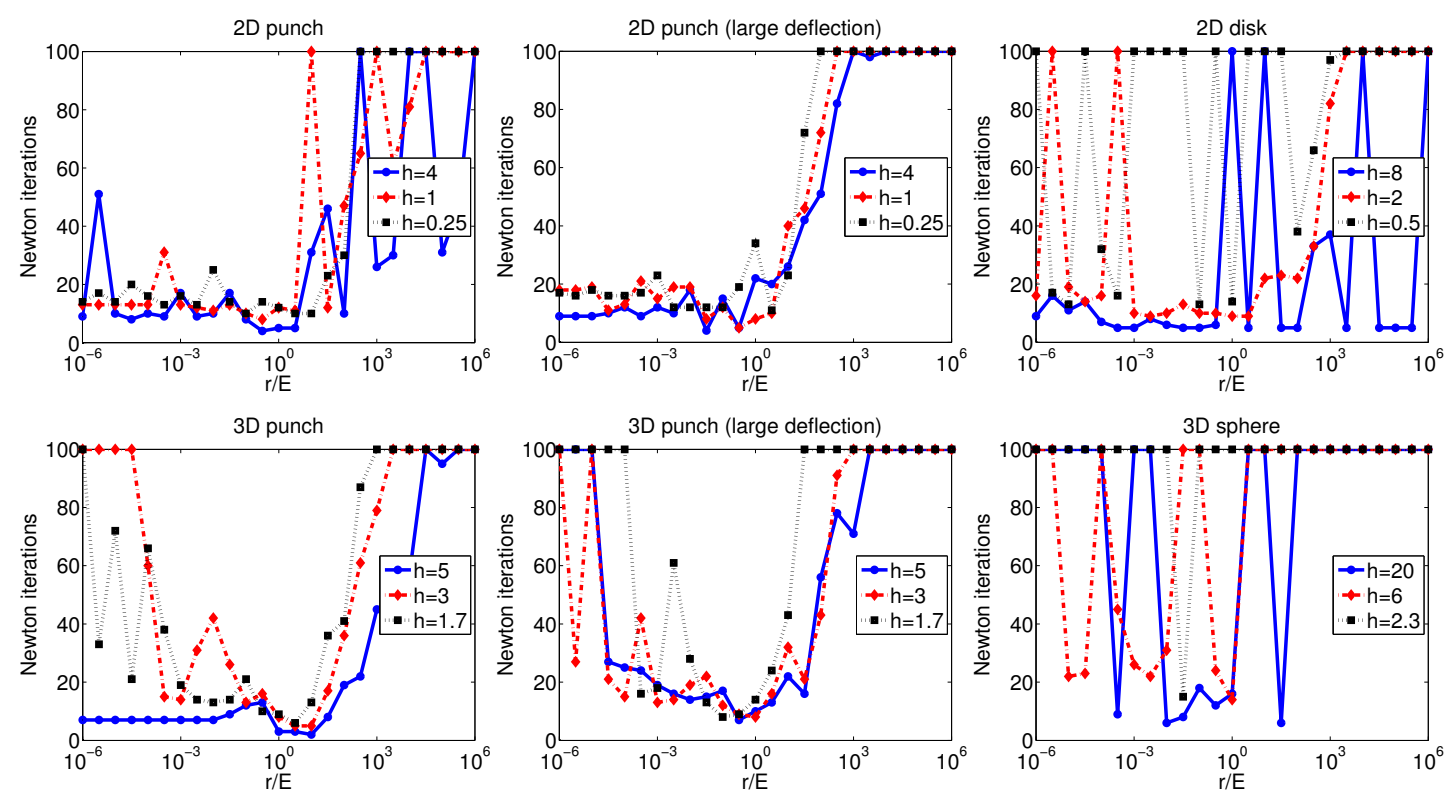

Figure 9: Number of iterations for Alart-Curnier generalized Newton's method with nodal contact with friction approximation. Symmetric version.
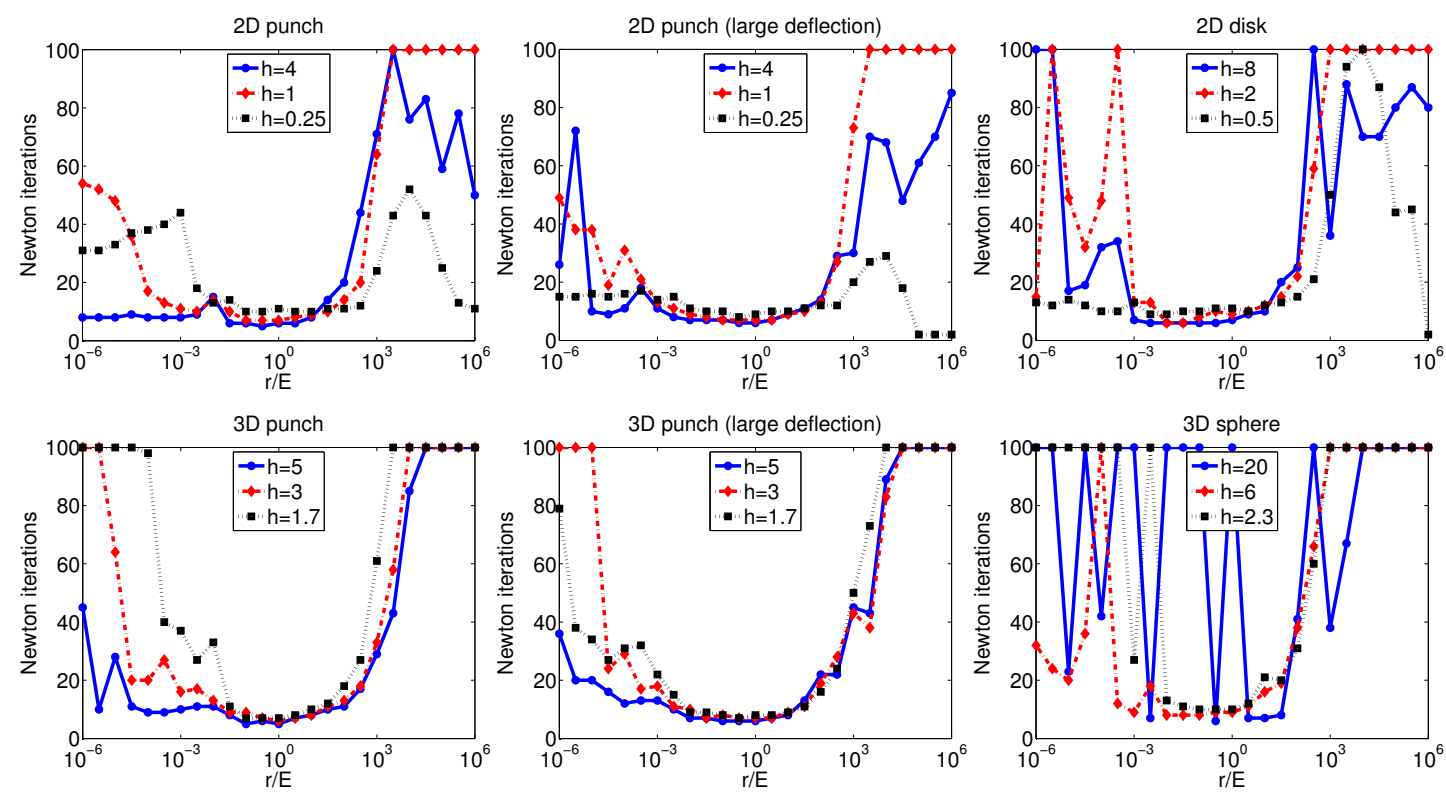

Figure 10: Number of iterations for Alart-Curnier generalized Newton's method with integral frictionless contact approximation. Unsymmetric version. 


\subsection{Integral approximation of the contact condition}

We turns now to the integral approximation of the contact with friction condition. The approximation of the less symmetric system (14) leads to the following system:

$$
\left\{\begin{array}{l}
a\left(u^{h}, v^{h}\right)=\ell\left(v^{h}\right)+\int_{\Gamma_{C}} \lambda_{N}^{h} v_{N}^{h} d \Gamma+\int_{\Gamma_{C}} \lambda_{T}^{h} \cdot v_{T}^{h} d \Gamma \quad \forall v^{h} \in V^{h}, \\
-\frac{1}{r} \int_{\Gamma_{C}}\left(\lambda_{N}^{h}+\left(\lambda_{N}^{h}-r\left(u_{N}^{h}-g\right)\right)_{-}\right) \mu_{N}^{h} d \Gamma=0 \quad \forall \mu^{h} \in W^{h}, \\
-\frac{1}{r} \int_{\Gamma_{C}}\left(\lambda_{T}^{h}-P_{B\left(0, \mathscr{F}\left(\lambda_{N}^{h}-r\left(u_{N}^{h}-g\right)\right)_{-}\right.}\left(\lambda_{T}^{h}-r u_{T}^{h}\right)\right) \cdot \mu_{T}^{h} d \Gamma=0 \quad \forall \mu^{h} \in W^{h} .
\end{array}\right.
$$

An existence result of a solution to Problem (15) for arbitrary $r>0$ and $\mathscr{F} \geq 0$ and a uniqueness result for both $r$ and $\mathscr{F}$ small enough can be found in [30].

The corresponding numerical experiments are presented in Fig. 10 and Fig. 11 for the cases without or with friction, respectively. The method is more sensitive to the value of the augmentation parameter in the frictionless case if one compare with Fig. 6 for the nodal approximation, except for the non-coercive case which behaves slightly better. For the case with friction, results are more similar. However, one may conclude that globally, Newton's method on the nodal discretization is more robust.
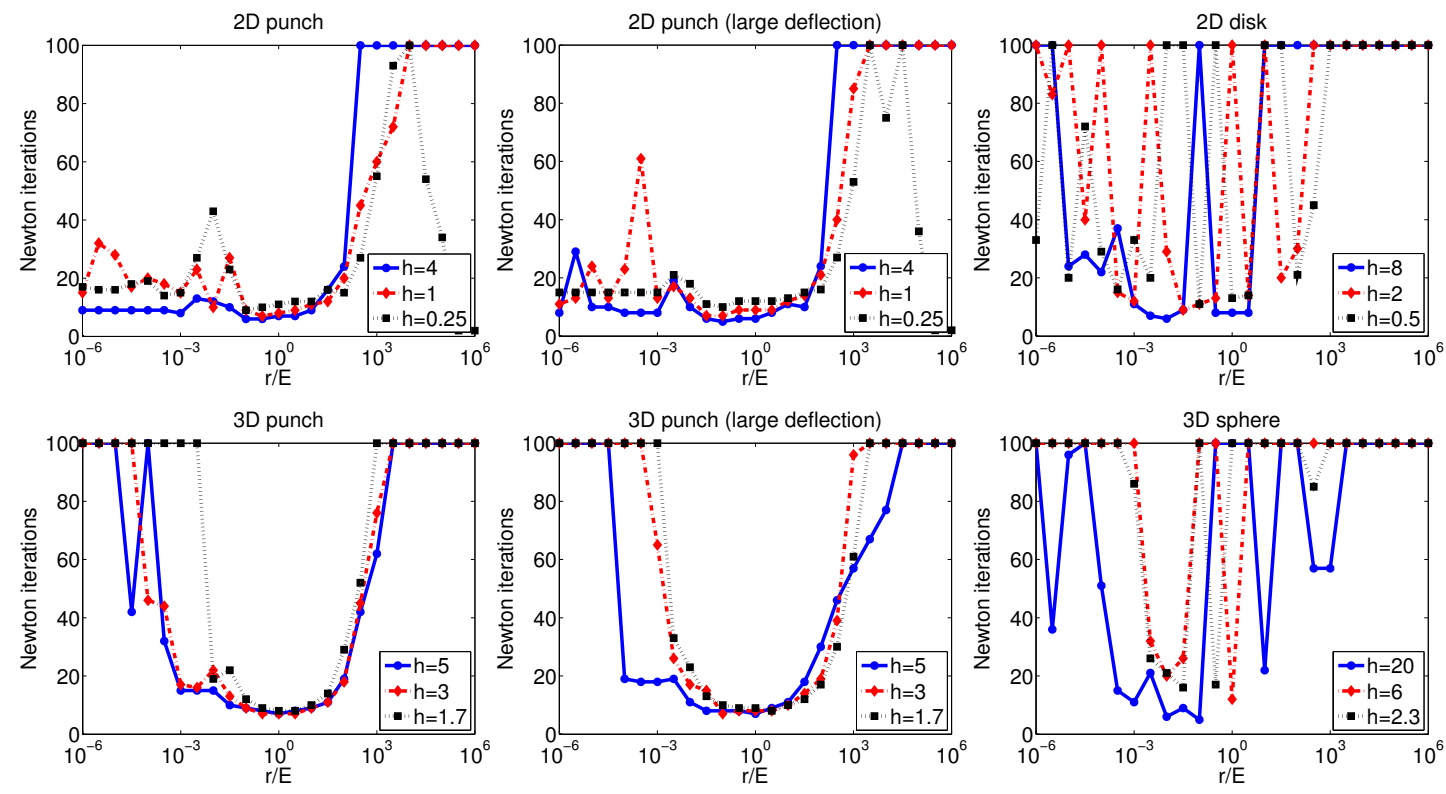

Figure 11: Number of iterations for Alart-Curnier generalized Newton's method with integral contact with friction approximation. Unsymmetric version.

Now, still with an integral approximation of the contact with friction condition, it is also 
possible to consider the more symmetric system:

$$
\left\{\begin{array}{l}
a\left(u^{h}, v^{h}\right)=\ell\left(v^{h}\right)+\int_{\Gamma_{C}}\left(\lambda_{N}^{h}-r\left(u_{N}^{h}-g\right)\right)_{-} v_{N}^{h} d \Gamma \\
\quad+\int_{\Gamma_{C}} P_{B\left(0,-\mathscr{F}\left(\lambda_{N}^{h}-r\left(u_{N}^{h}-g\right)\right)_{-}\right)}\left(\lambda_{T}^{h}-r u_{T}^{h}\right) \cdot v_{T}^{h} d \Gamma \quad \forall v^{h} \in V^{h}, \\
-\frac{1}{r} \int_{\Gamma_{C}}\left(\lambda_{N}^{h}+\left(\lambda_{N}^{h}-r\left(u_{N}^{h}-g\right)\right)_{-}\right) \mu_{N}^{h} d \Gamma=0 \quad \forall \mu^{h} \in W^{h}, \\
-\frac{1}{r} \int_{\Gamma_{C}}\left(\lambda_{T}^{h}-P_{B\left(0, \mathscr{F}\left(\lambda_{N}^{h}-r\left(u_{N}^{h}-g\right)\right)_{-}\right)}\left(\lambda_{T}^{h}-r u_{T}^{h}\right)\right) \cdot \mu_{T}^{h} d \Gamma=0 \quad \forall \mu^{h} \in W^{h} .
\end{array}\right.
$$

The number of Newton iterations are shown in Fig. 12 for the frictional case. The convergence is very similar to the one of the unsymmetric version with only a slight degradation in $3 \mathrm{D}$ cases. The frictionless case being similar, the graphs are not shown for shortness.
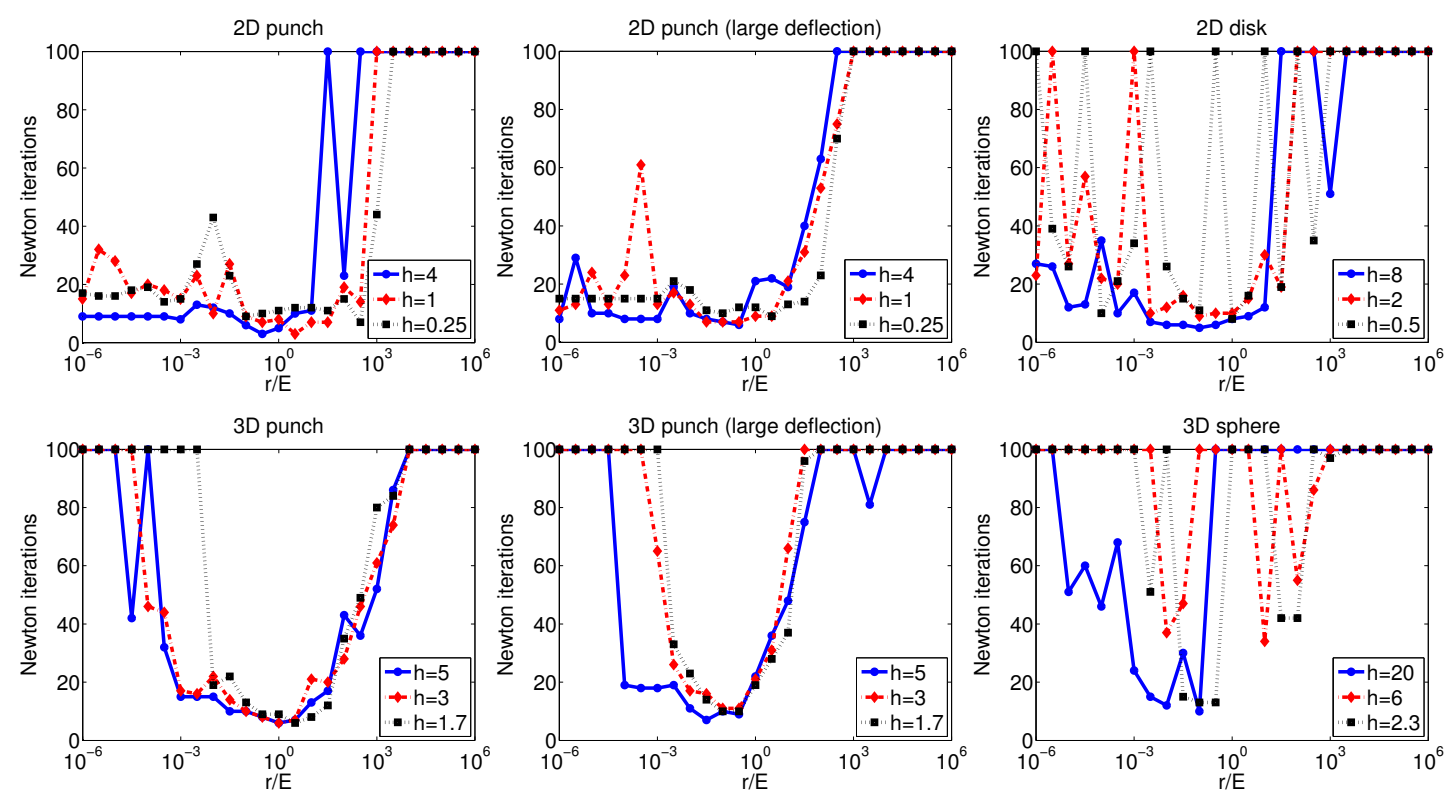

Figure 12: Number of iterations for Alart-Curnier generalized Newton's method with integral contact with friction approximation. Symmetric version.

\section{Simo-Laursen method}

\subsection{The original method}

The Simo-Laursen method corresponds to an Uzawa algorithm (in the case of Tresca friction). Still for shortness, only the integral approximation of contact is considered. The nodal approximation give similar results. The first step, for a contact stress $\lambda^{h, i}$ given is to find $u^{h, i+1}$ solution to

$$
\begin{aligned}
& a\left(u^{h, i+1}, v^{h}\right)=\ell\left(v^{h}\right)+\int_{\Gamma_{C}}\left(\lambda_{N}^{h, i}-r\left(u_{N}^{h, i+1}-g\right)\right)_{-} v_{N}^{h} d \Gamma \\
& \quad+\int_{\Gamma_{C}} P_{B\left(0,-\mathscr{F}\left(\lambda_{N}^{h, i}-r\left(u_{N}^{h, i+1}-g\right)\right)_{-}\right)}\left(\lambda_{T}^{h, i}-r u_{T}^{h, i+1}\right) \cdot v_{T}^{h} d \Gamma \quad \forall v^{h} \in V^{h} .
\end{aligned}
$$



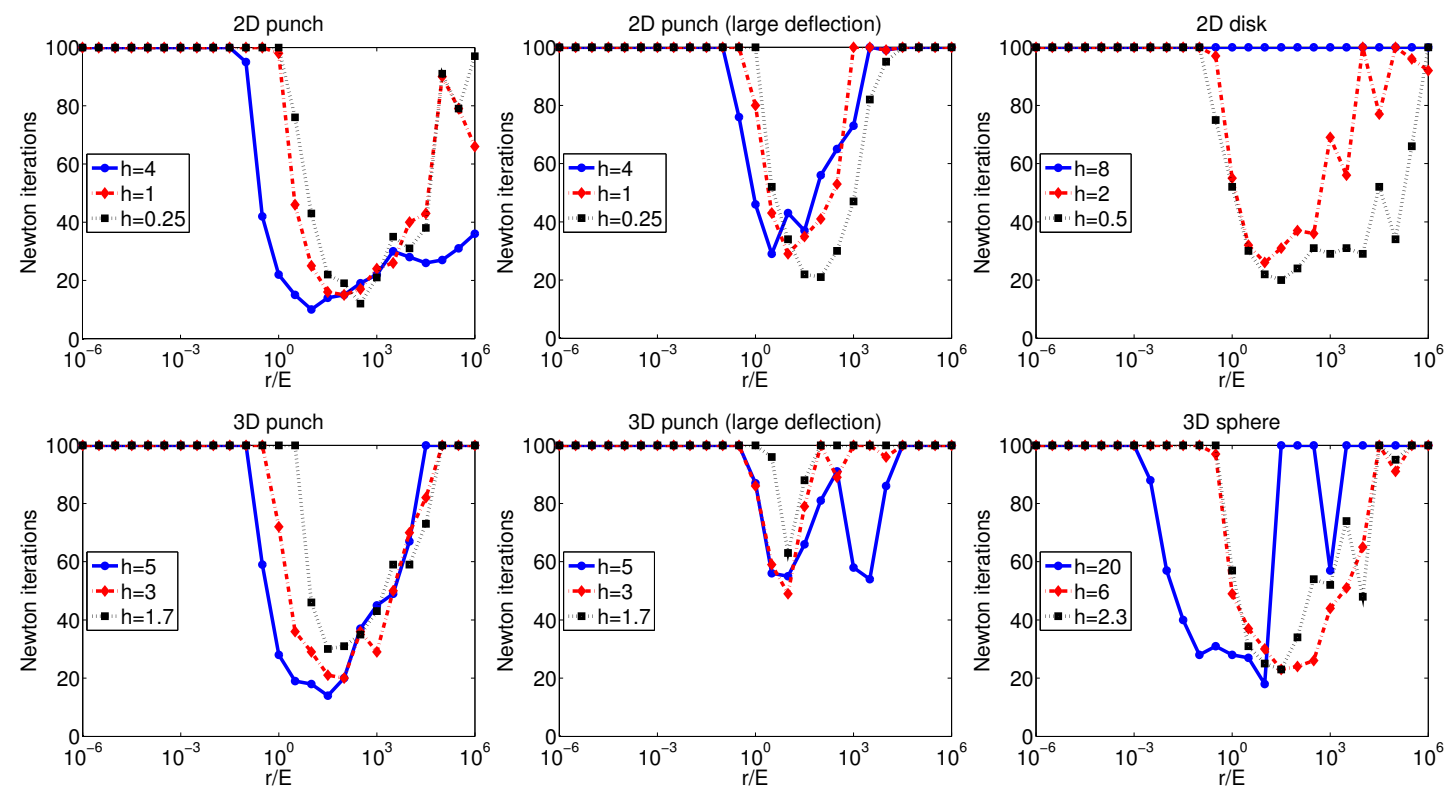

Figure 13: Cumulative number of Newton iterations for the Simo-Laursen method with integral frictionless contact approximation.
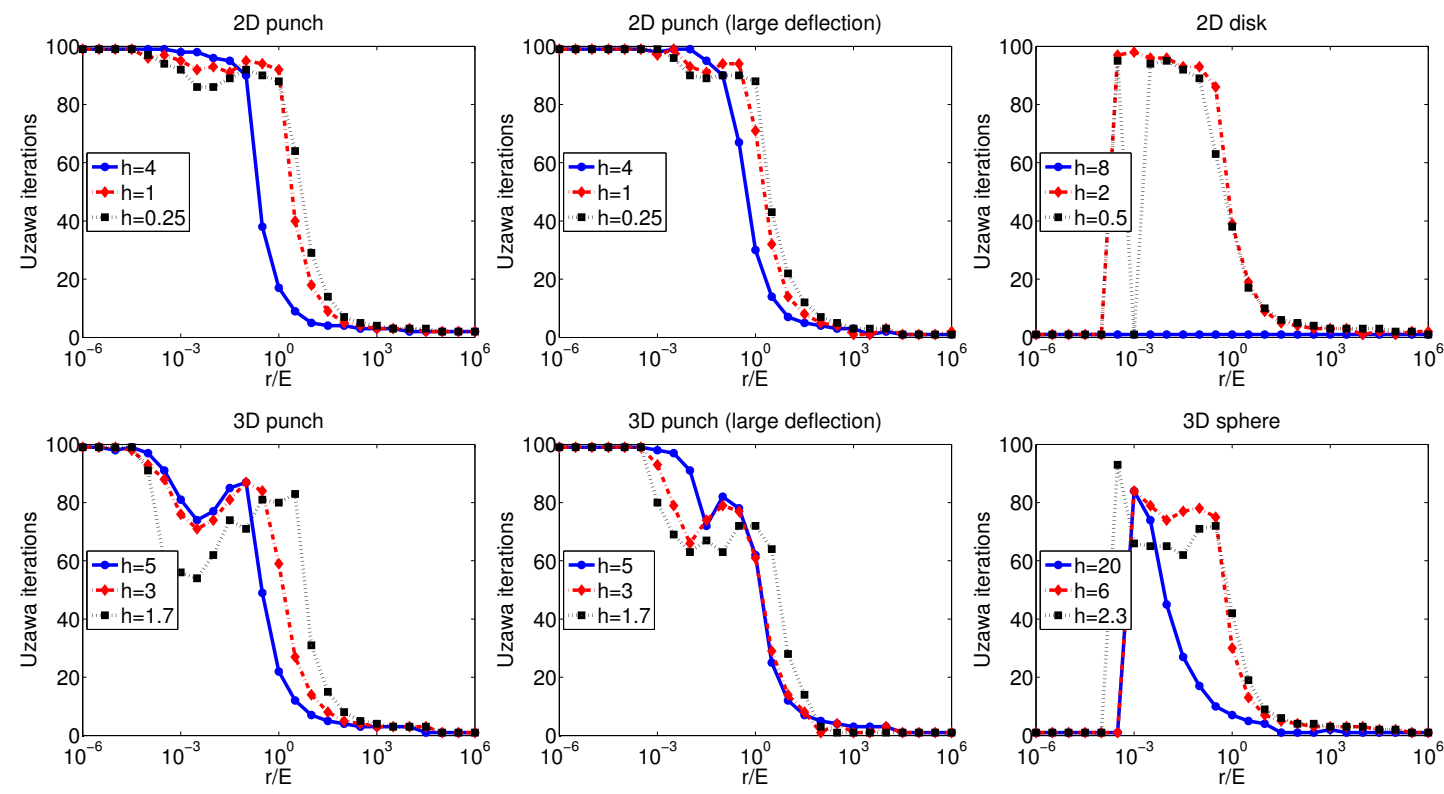

Figure 14: Number of Uzawa iterations for the Simo-Laursen method with integral frictionless contact approximation.

This first step is solved with a generalized Newton's method. Then, the second step consists 
in computing $\lambda^{h, i+1}$ thanks to

$$
\begin{aligned}
& \int_{\Gamma_{C}}\left(\lambda_{N}^{h, i+1}+\left(\lambda_{N}^{h, i}-r\left(u_{N}^{h, i+1}-g\right)\right)_{-}\right) \mu_{N}^{h} d \Gamma=0 \quad \forall \mu^{h} \in W^{h}, \\
& \int_{\Gamma_{C}}\left(\lambda_{T}^{h, i+1}-P_{B\left(0,-\mathscr{F}\left(\lambda_{N}^{h, i}-r\left(u_{N}^{h, i+1}-g\right)\right)_{-}\right)}\left(\lambda_{T}^{h, i}-r u_{T}^{h, i+1}\right)\right) \cdot \mu_{T}^{h, i} d \Gamma=0 \quad \forall \mu^{h} \in W^{h} .
\end{aligned}
$$

In the frictionless case, Fig. 13 and Fig. 14 present the total number of Newton iterations (summed over the Uzawa iterations) and the number of Uzawa iterations, respectively. The analysis of the two series of graphs indicates that the number of Newton iterations inside an Uzawa iteration increases with the augmentation parameter $r$. This is a classical result when the contact condition is approached by penalization which is found here certainly because of the similarity between this formulation and the penalized one. Conversely, the largest is the augmentation parameter, the fastest the Uzawa method converges (on the graphs, remember that the algorithm is stopped after 100 cumulated Newton iterations, whatever the number of Uzawa iterations). The main difficulty of the Simo-Laursen method is to find the good compromise to allow both a reasonable number of iterations for Newton's method and for the Uzawa algorithm. Fig. 13 and Fig. 14 shows that this may not be easy. The difficulty of the convergence of Newton's method for the penalized problem is addressed in some works such as [44] where the contact condition is modified in the first Newton iterations in order to facilitate the convergence. These techniques can also be applied to the Simo-Laursen method. However, this difficulty is a drawback of the method. The same conclusion can be applied to the case with friction on analyzing Fig. 15. Note also the significant sensitivity to the size of the load and the shift of the optimal augmentation parameter when the mesh is refined which have not been observed for Alart-Curnier method.
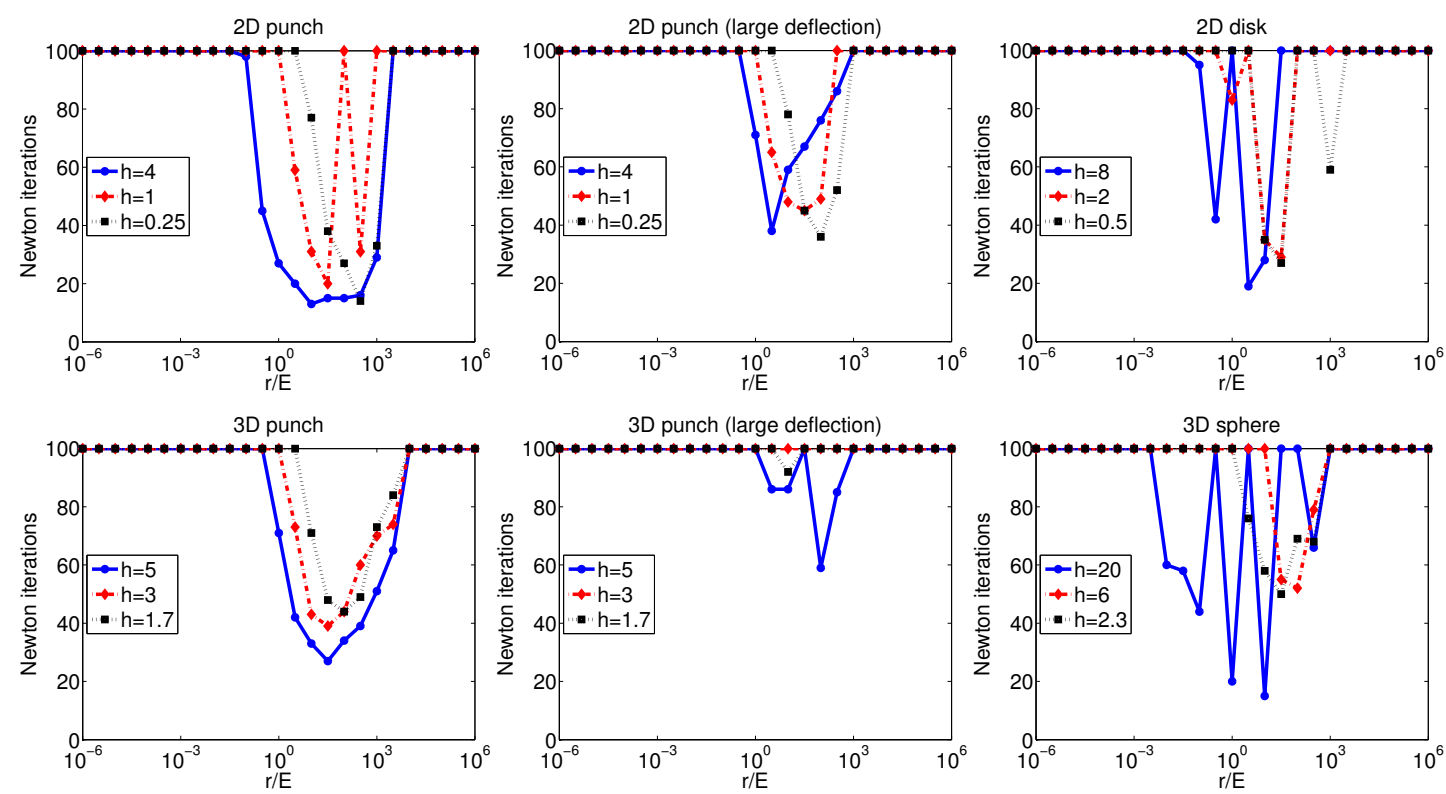

Figure 15: Cumulative number of Newton iterations for the Simo-Laursen method with integral contact with friction approximation. 


\subsection{Using De Saxcé's projection}

Another possibility, coming from De Saxcé bipotential theory (see $[13,30])$ is to replace the two separate projections (10) and (11) for the contact and friction conditions by the following single one:

$$
\left.\lambda^{h}=P_{\Lambda_{\mathscr{F}}}\left(\lambda^{h}-r\left(\left(u_{N}^{h}-g\right)-\mathscr{F}\left|u_{T}^{h}\right|\right) n_{0}+u_{T}^{h}\right)\right),
$$

where $\Lambda_{\mathscr{F}}$ is the friction cone defined by

$$
\Lambda_{\mathscr{F}}=\left\{\lambda \in \mathbb{R}^{d}:\left|\lambda_{T}\right| \leq-\mathscr{F} \lambda_{N}\right\}
$$

and $P_{\Lambda_{\mathscr{F}}}$ the orthogonal projection which reads

$$
P_{\Lambda_{\mathscr{F}}}(\lambda)=\left\{\begin{array}{l}
0 \text { if } \mathscr{F}\left|\lambda_{T}\right| \leq \lambda_{N}, \\
\lambda \text { if }\left|\lambda_{T}\right| \leq-\mathscr{F} \lambda_{N}, \\
\frac{\lambda_{N}-\mathscr{F}\left|\lambda_{T}\right|}{\mathscr{F}^{2}+1}\left(n_{0}-\mathscr{F} \frac{\lambda_{T}}{\left|\lambda_{T}\right|}\right) \text { otherwise. }
\end{array}\right.
$$

Using this unique projection, the first step of Uzawa's algorithm corresponds now to find $u^{h, i+1}$ solution to

$$
\left.a\left(u^{h, i+1}, v^{h}\right)=\ell\left(v^{h}\right)+\int_{\Gamma_{C}} P_{\Lambda_{\mathscr{F}}}\left(\lambda^{h, i}-r\left(\left(u_{N}^{h, i+1}-g\right)-\mathscr{F}\left|u_{T}^{h, i+1}\right|\right) n_{0}+u_{T}^{h, i+1}\right)\right) \cdot v d \Gamma,
$$

for a given $\lambda^{h, i}$, and the second step corresponds to compute $\lambda^{h, i+1}$ thanks to

$$
\int_{\Gamma_{C}}\left(\lambda^{h, i+1}-P_{\Lambda_{\mathscr{F}}}\left(\lambda^{h, i}-r\left(\left(u_{N}^{h, i+1}-g\right)-\mathscr{F}\left|u_{T}^{h, i+1}\right|\right) n_{0}+u_{T}^{h, i+1}\right)\right) \cdot \mu d \Gamma=0 \quad \forall \mu^{h} \in W^{h} .
$$
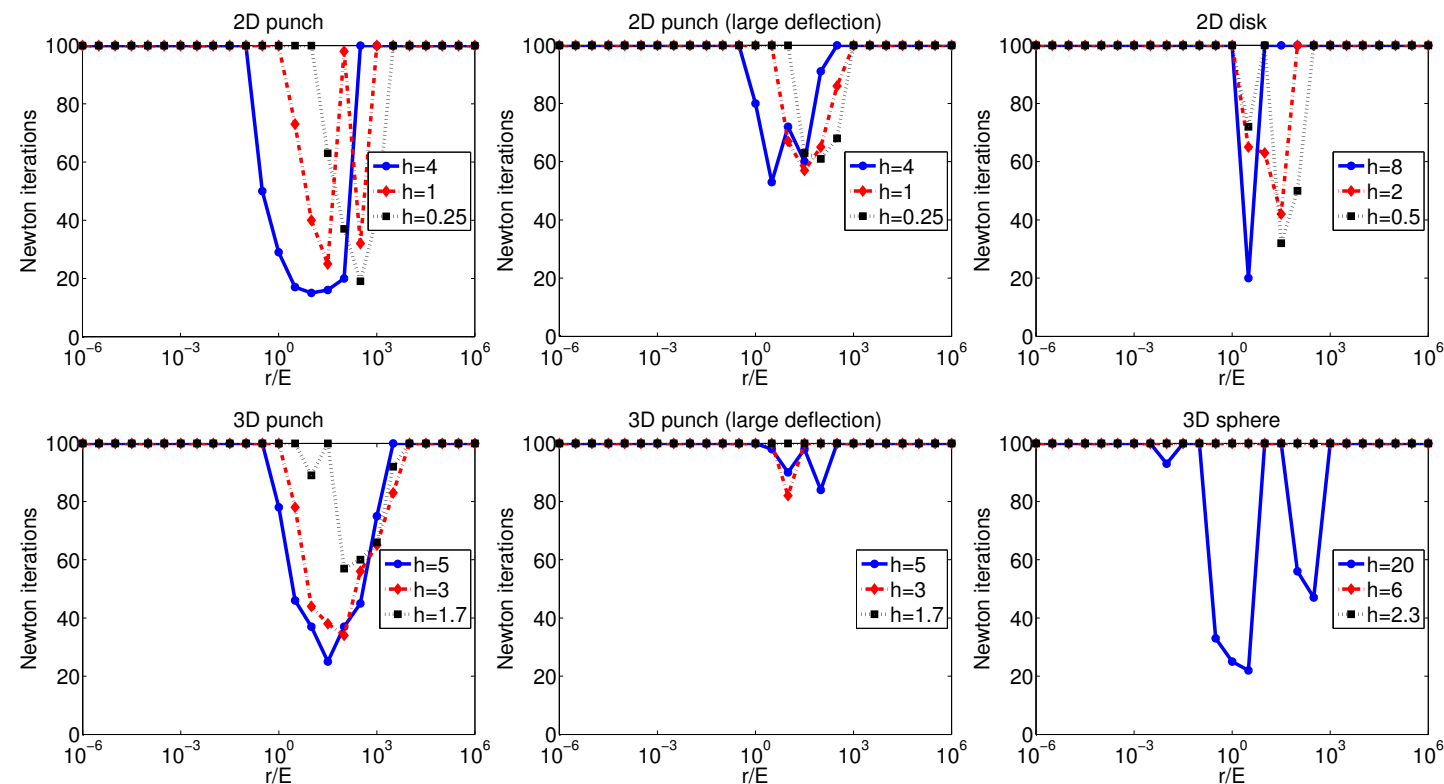

Figure 16: Cumulative number of Newton iterations for the Simo-Laursen method using De Saxcé projection and with integral contact with friction approximation. 
A significant aspect of this formulation is the non-differentiability of the term $\left|u_{T}^{h, i+1}\right|$ occurring for $u_{T}^{h, i+1}=0$ which corresponds of course to non-improbable situations (since it corresponds to a sticking solution). One may consider that this could affect the convergence of Newton's method since order two of convergence can be lost at non-differentiable points (see [34, 42]). Such a degradation of the convergence is not clearly visible on graphs of Fig. 16 which represent the case with friction (De Saxcé's projection has of course no interest in the frictionless case). However, we can conclude that the use of De Saxcé's projection do not improve the convergence here.

Note also that the use of De Saxcé's projection has also been tested on Alart-Curnier generalized Newton's method with also very few differences on the number of iterations for convergence. Numerical results are again not presented for shortness of the paper.

\section{Nitsche's method adapted to contact with friction}

Original Nitsche's method [33] allows to prescribe a Dirichlet condition on a boundary in a consistent way without the use of a multiplier. The extension to the contact condition is a recent concern. In [18] an extension to bilateral (persistent) contact is proposed. In [43] the method is extended to large strain bilateral frictionless contact. In [9] the method is extended to unilateral contact and a numerical analysis is performed which shows the optimality of the method. An interesting result of [9] is the full optimality of the a priori error estimate without any additional assumption on the solution than having the appropriate regularity. This is all the more remarkable that this is the first approximation of contact condition in elasticity for wich an optimal a priori error estimate has been proven. See for instance [21] and the references there in for a priori error estimates of standard approximation of contact.

\subsection{Symmetric version of Nitsche's method}

We present here the Nitsche-based method presented in [9] and extended to Coulomb friction. For frictionless problem, the tangent system is symmetric (of course there is an unavoidable unsymmetric term when Coulomb friction is considered). In the formalism of the rest of the paper, the corresponding problem reads as

$$
\left\{\begin{array}{l}
\text { Find } u^{h} \in V^{h} \text { satisfying } \\
a\left(u^{h}, v^{h}\right)-\frac{1}{r} \int_{\Gamma_{C}}(\sigma(u) n) \cdot(\sigma(v) n) d \Gamma-\frac{1}{r} \int_{\Gamma_{C}}\left(\sigma_{N}\left(u^{h}\right)-r\left(u_{N}^{h}-g\right)\right)_{-}\left(\sigma_{N}(v)-r v_{N}\right) d \Gamma \\
+\frac{1}{r} \int_{\Gamma_{C}} P_{B\left(0, \mathscr{F}\left(\sigma_{N}\left(u^{h}\right)-r\left(u_{N}^{h}-g\right)\right)_{-}\right)}\left(\sigma_{T}\left(u^{h}\right)-r u_{T}^{h}\right) \cdot\left(\sigma_{T}\left(v^{h}\right)-r v_{T}^{h}\right) d \Gamma=l\left(v^{h}\right), \quad \forall v \in V^{h} .
\end{array}\right.
$$

There is at least two main advantages of Nitsche's method. It leads to a weak formulation which consists in an equation (not an inequation) on which a generalized Newton's method can be directly applied. Similarly to a penalized formulation, the only unknown is the displacement field. The method is consistent which means that the non-penetration condition holds for any parameter $r>0$. However, the parameter $r$ should be chosen sufficiently large to keep the coercivity of the tangent matrix (see [9]). In fact the resolution of Problem (17) is very similar to the resolution of the first step of Simo-Laursen method presented in the previous section. A main difference is that the terms $\lambda_{N}$ and $\lambda_{T}$ are replaced by $\sigma_{N}\left(u^{h}\right)$ and $\sigma_{T}\left(u^{h}\right)$, respectively, and there is an additional term ensuring the consistency. Of course, the advantage compared to Simo-Laursen method is that no Uzawa iteration is needed. A small disadvantage of the 
method is the need of the material constitutive law for the computation of $\sigma(u) n$ which makes the implementation dependent on it.
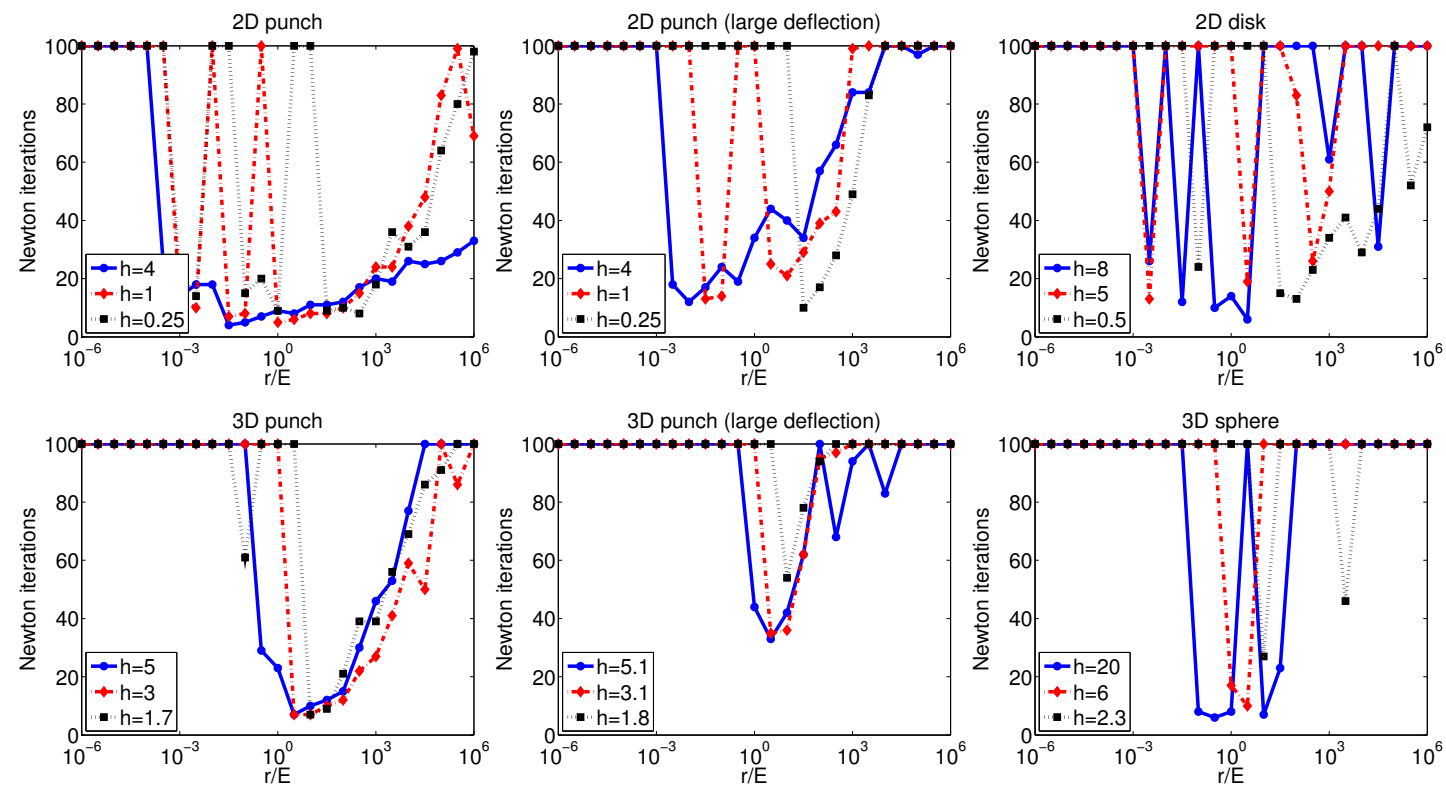

Figure 17: Number of Newton iterations for symmetric Nitsche's method adapted to frictionless contact and an integral approximation.
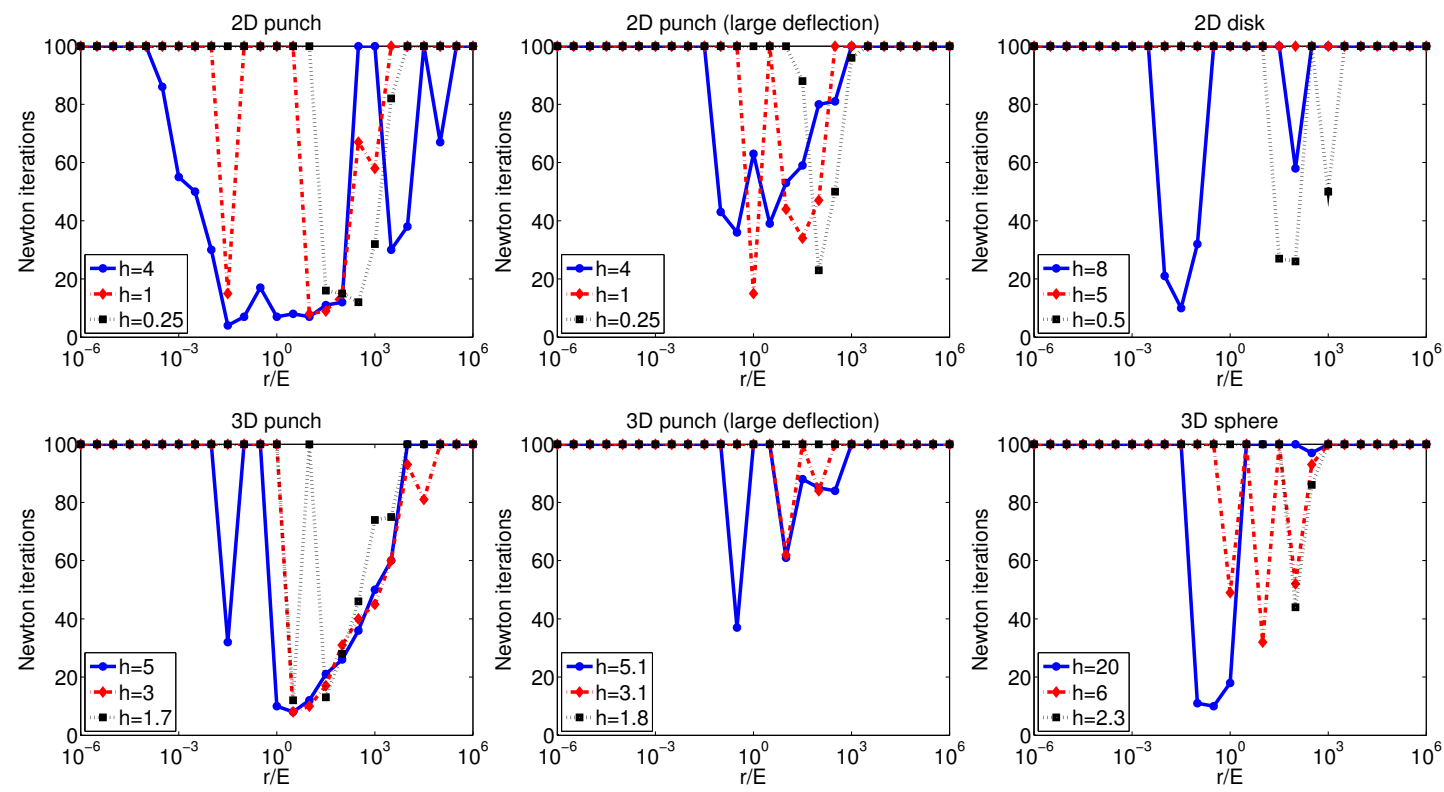

Figure 18: Number of Newton iterations for symmetric Nitsche's method adapted to contact with friction and an integral approximation.

The number of iterations of the corresponding generalized Newton's method are presented in Fig. 17 for the frictionless case and Fig. 18 for the case with friction. The result are very similar 
to the ones for the Simo-Laursen method with the same kind of difficulties. On may think that a strategy like the one proposed in [44] may be applied to improve the convergence.

Additionally here, when the parameter $r$ is too small and the coercivity is lost, the Newton algorithm may converge toward a non-physical solution. This minimal parameter can be estimated by the computation of the smallest eigenvalue of the tangent matrix. The minimal parameter $r$ was found to be about $15 E$ for the largest meshes and $60 E$ for the finest one in the presented numerical results.

\subsection{An unsymmetric version of Nitsche's method}

A rather simpler version can be obtained from (17) by remarking that the term $\sigma(v) n$ is not necessary for the consistency of the method. The following formulation is then obtained:

$$
\left\{\begin{array}{l}
\text { Find } u^{h} \in V^{h} \text { satisfying } \\
a\left(u^{h}, v^{h}\right)+\int_{\Gamma_{C}}\left(\sigma_{N}\left(u^{h}\right)-r\left(u_{N}^{h}-g\right)\right)_{-} v_{N} d \Gamma \\
-\int_{\Gamma_{C}} P_{B\left(0, \mathscr{F}\left(\sigma_{N}\left(u^{h}\right)-r\left(u_{N}^{h}-g\right)\right)_{-}\right)}\left(\sigma_{T}\left(u^{h}\right)-r u_{T}^{h}\right) \cdot v_{T}^{h} d \Gamma=l\left(v^{h}\right), \quad \forall v \in V^{h} .
\end{array}\right.
$$

Compared to (17) this formulation is non-symmetric even in the frictionless case. The number of iterations of the corresponding generalized Newton's method are presented in Fig. 19 for the frictionless case and Fig. 20 for the case with friction. The convergence of Newton's method is greatly improved in comparison to the symmetric version. Another significant advantage is that the method seems to converges toward a physical solution for much smaller values of the parameter $r$. As a conclusion, for contact with friction problems, unsymmetric Nitsche's method behave much better than the symmetric one. This method is also discussed in [10].
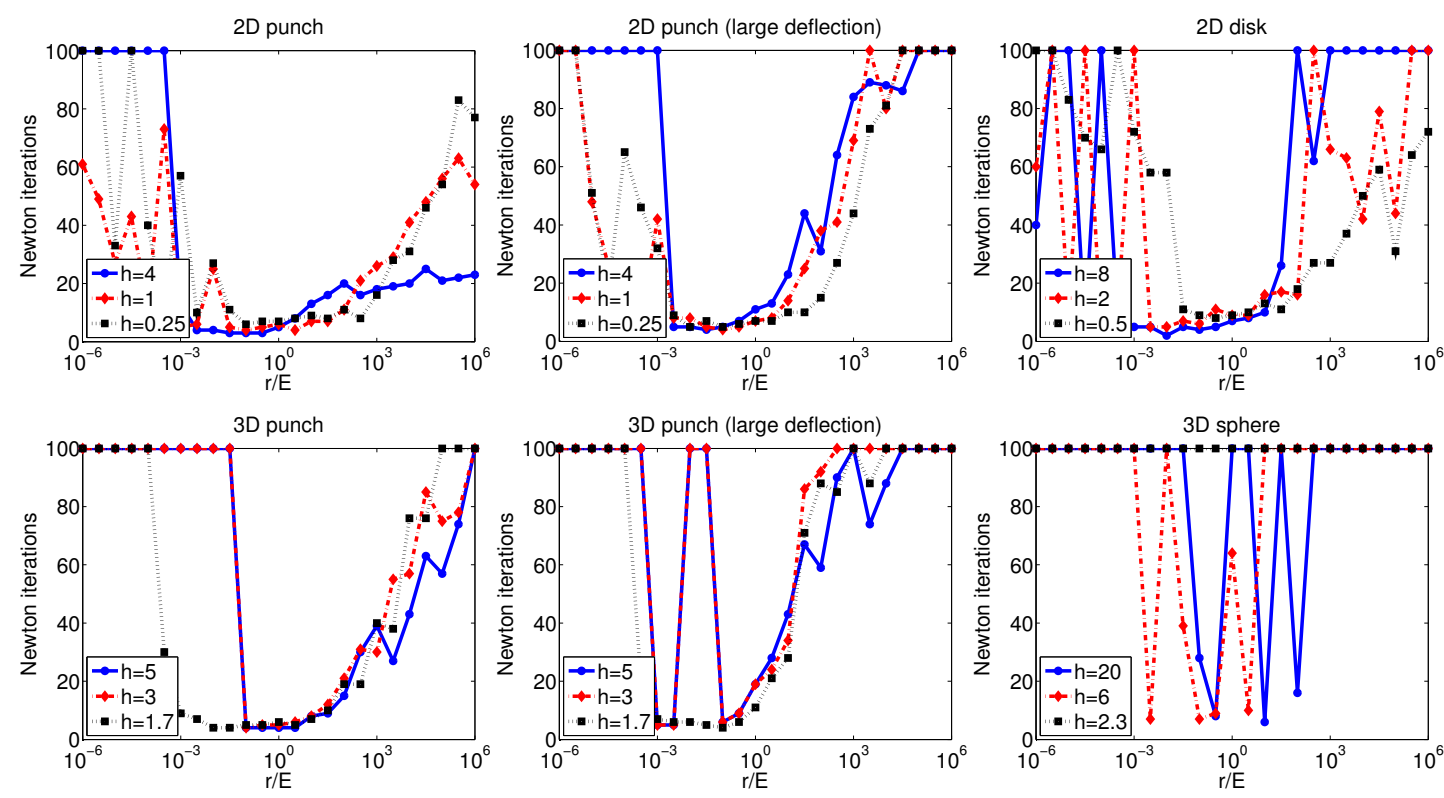

Figure 19: Number of Newton iterations for unsymmetric Nitsche's method adapted to frictionless contact and an integral approximation. 

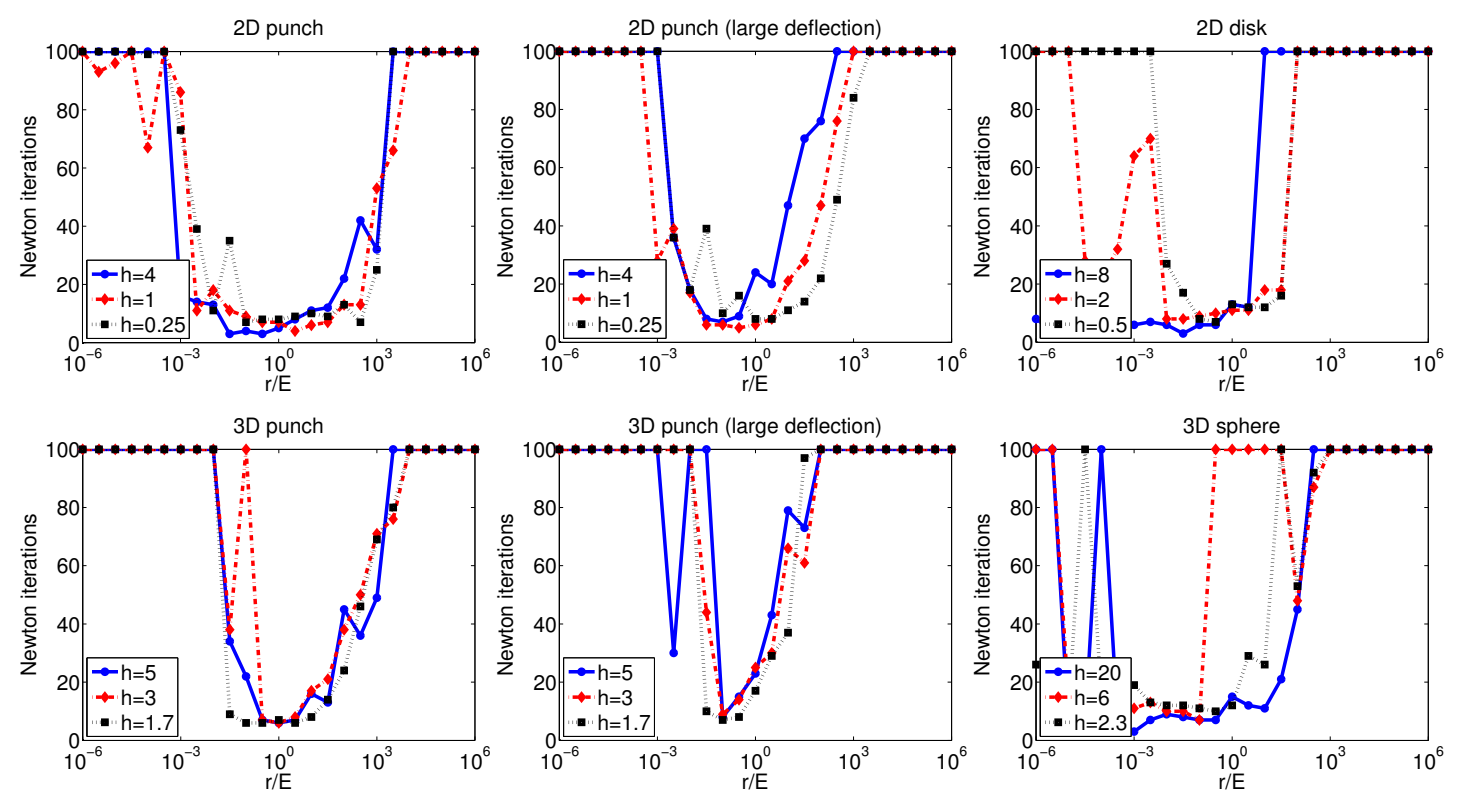

Figure 20: Number of Newton iterations for unsymmetric Nitsche's method adapted to contact with friction and an integral approximation.

\section{A new generalized Newton's method based on augmented mul- tipliers}

Unlike previously presented methods, the method proposed here does not derive from an augmented Lagrangian. The principle is to consider an auxiliary variable instead of the multiplier representing the contact forces. For the contact condition, let us consider $\xi_{N}$ a real variable whose negative part will store the normal gap and whose positive part will store the contact pressure:

$$
\left(\xi_{N}\right)_{+}=r\left(g-u_{N}\right), \quad\left(\xi_{N}\right)_{-}=-\lambda_{N},
$$

where $r>0$ represent a scaling coefficient which allows the variable $\xi$ to be described with an homogeneous unit $\left(\mathrm{N} / \mathrm{m}^{2}\right)$. The advantage of this auxiliary variable, the so-called augmented multiplier, is that the complementarity relation is automatically satisfied since $\xi_{N}$ is either negative or positive. Similarly, for the friction condition, one can consider the augmented multiplier $\xi_{T} \in \mathbb{R}^{d-1}$ satisfying

$$
\begin{aligned}
P_{\bar{B}\left(0, \mathscr{F}\left(\xi_{N}\right)_{-}\right)}\left(\xi_{T}\right) & =\lambda_{T}, \\
\xi_{T}-P_{\bar{B}\left(0, \mathscr{F}\left(\xi_{N}\right)_{-}\right)}\left(\xi_{T}\right) & =-r u_{T} .
\end{aligned}
$$

There is some similarities with the formulation of Ben Dhia and Zarroug $[6,5]$ which is however rather different since, for instance, an additional discontinuous function with an active set strategy is used. 


\subsection{Nodal approximation of the contact condition}

Introducing some augmented multipliers for each finite element nodes leads to the following system:

$$
\left\{\begin{array}{l}
a\left(u^{h}, v^{h}\right)=\ell\left(v^{h}\right)-\sum_{a \in \mathcal{N}_{\Gamma_{C}}}\left(\xi_{N}^{a}\right)_{-} v_{N}^{h}(a)+P_{\bar{B}\left(0, \mathscr{F}\left(\xi_{N}\right)_{-}\right)}\left(\xi_{T}^{a}\right) \cdot v_{T}^{h}(a) \quad \forall v^{h} \in V^{h}, \\
-\frac{1}{r}\left(r\left(u_{N}^{h}(a)-g(a)\right)+\left(\xi_{N}^{a}\right)_{+}\right)=0, \quad \forall a \in \mathcal{N}_{\Gamma_{C}}, \\
-\frac{1}{r}\left(r u_{T}^{h}(a)+\xi_{T}^{a}-P_{\bar{B}\left(0,-\mathscr{F}\left(\xi_{N}\right)_{-}\right)}\left(\xi_{T}^{a}\right)\right)=0, \quad \forall a \in \mathcal{N}_{\Gamma_{C}} .
\end{array}\right.
$$

The discrete problem (19) is still equivalent to the standard nodal contact approximation (8). Thus, problems (8) and (19) share the same properties for existence and uniqueness of the solution.

The numerical experiments are presented in Fig. 21 and Fig. 22 for the contact without and with friction respectively. In the frictionless case, one sees that the number of iterations is nearly independent of the parameter $r$ (except for the 3D cases and a large $r$ ). The novelty is that the method works also without difficulty for the non-coercive case. The situation is less favorable in the frictional case, especially for the 3D cases where the range of parameter $r$ over which the number of Newton iterations is less than 20 is rather narrower compared to Fig. 7 for the Alart-Curnier approach (except for the non-coercive cases).
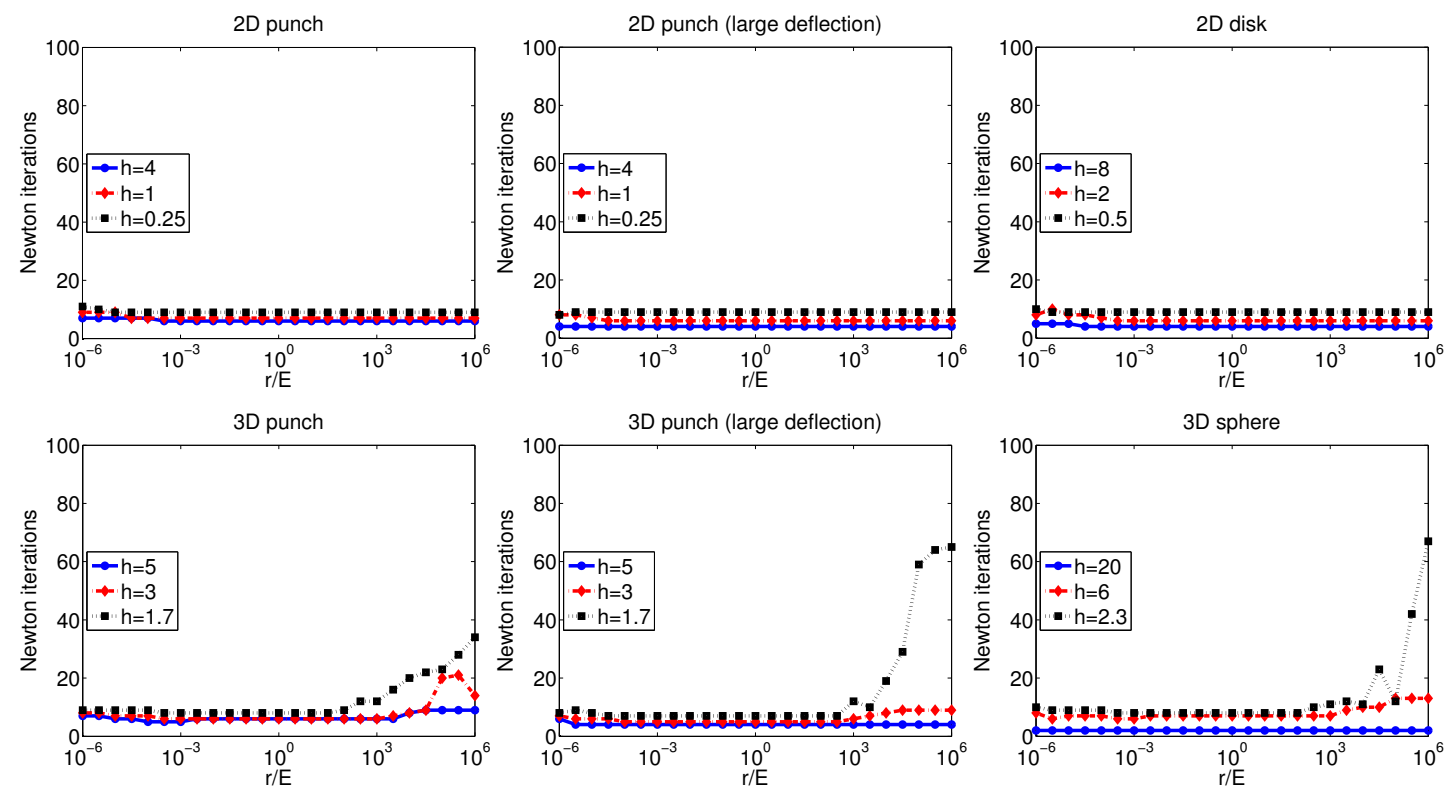

Figure 21: Number of iterations for the new Newton's method with nodal frictionless contact approximation. 

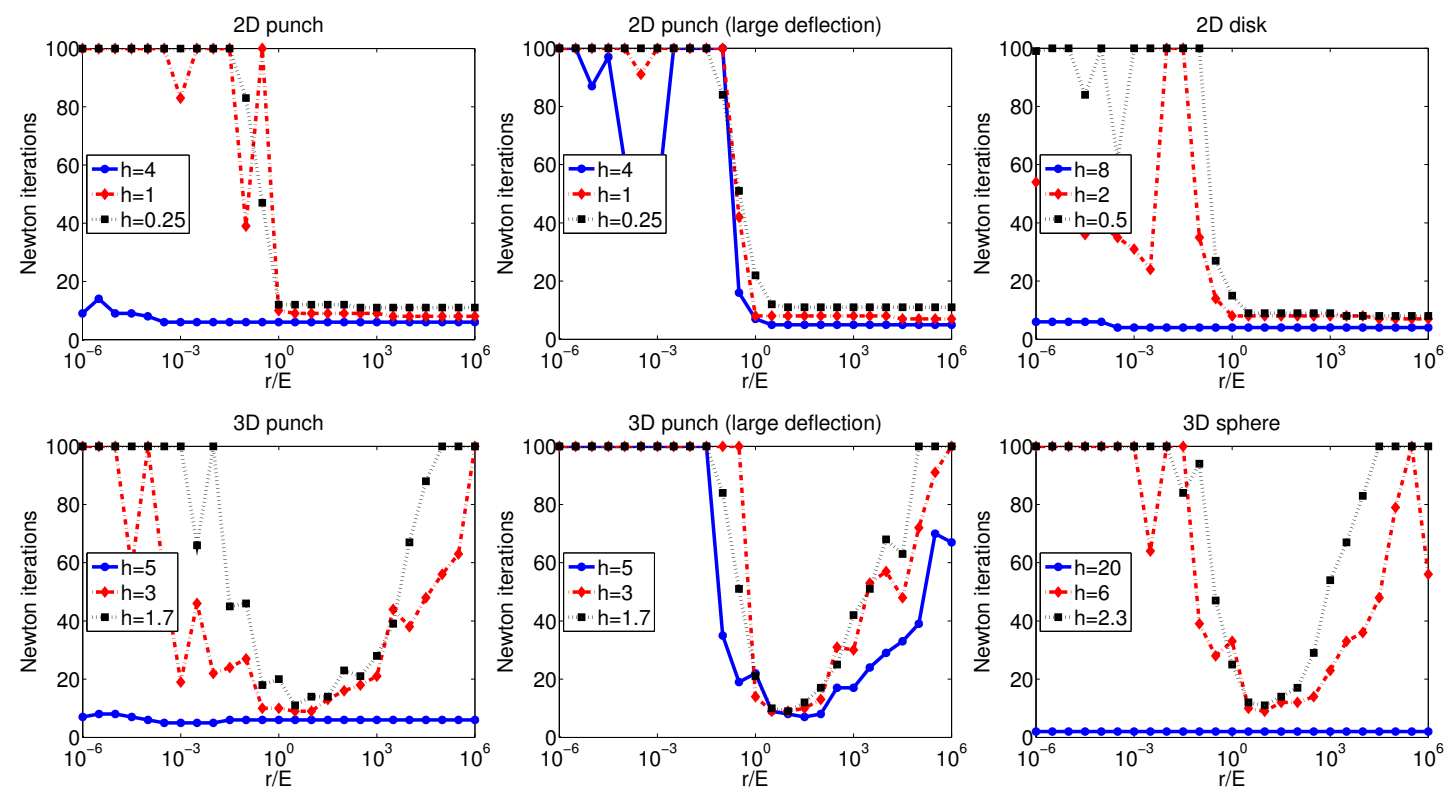

Figure 22: Number of iterations for the new Newton's method with nodal contact with friction approximation.
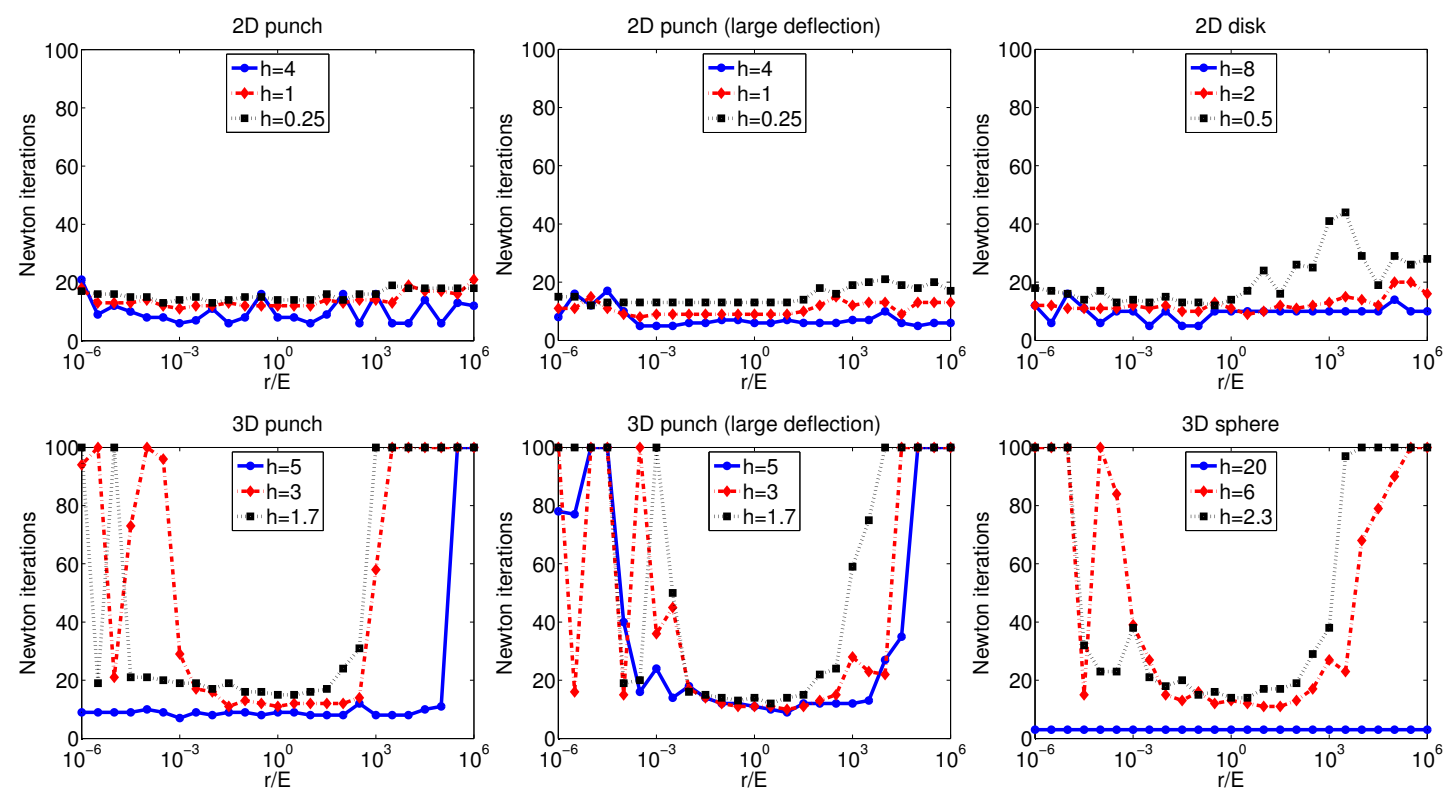

Figure 23: Number of iterations for the new Newton's method using De Saxcé projection and with nodal contact with friction approximation. 


\subsection{Nodal approximation of the contact condition with De Saxcé's projection}

The same principle can be applied to De Saxcé's formulation of contact with friction (16) introducing $\xi \in \mathbb{R}^{d}$ an augmented multiplier satisfying

$$
\begin{aligned}
P_{\Lambda_{\mathscr{F}}}(\xi) & =\lambda, \\
P_{\Lambda_{\mathscr{F}}}(\xi)-\xi & =-\left(r\left(u_{N}-g\right)-\mathscr{F}\left|u_{T}\right|\right) n_{0}-r u_{T} .
\end{aligned}
$$

The numerical experiments made on the corresponding discrete problem with nodal approximation of contact and friction condition is shown in Fig. 23. This method is the less sensitive to the parameter $r$ compared to all other tested methods when friction is considered, especially for $2 \mathrm{D}$ cases. The results are also very satisfactory for $3 \mathrm{D}$ and non-coercive cases.

\subsection{Integral approximation of the contact condition}

The counterpart of the nodal formulation (19) in term of integral approximation of the contact condition reads as follows:

$$
\left\{\begin{array}{l}
a\left(u^{h}, v^{h}\right)=\ell\left(v^{h}\right)-\int_{\Gamma_{C}}\left(\xi_{N}^{h}\right)_{-} v_{N}^{h} d \Gamma+\int_{\Gamma_{C}} P_{B\left(0, \mathscr{F}\left(\xi_{N}^{h}\right)_{-}\right)}\left(\xi_{T}^{h}\right) \cdot v_{T}^{h} d \Gamma \quad \forall v^{h} \in V^{h}, \\
-\int_{\Gamma_{C}}\left(\left(u_{N}^{h}-g\right)+\frac{1}{r}\left(\xi_{N}^{h}\right)_{+}\right) \mu_{N}^{h} d \Gamma=0 \quad \forall \mu^{h} \in W^{h}, \\
-\int_{\Gamma_{C}}\left(u_{T}^{h}+\frac{1}{r} \xi_{T}^{h}-\frac{1}{r} P_{B\left(0, \mathscr{F}\left(\xi_{N}^{h}\right)_{-}\right)}\left(\xi_{T}^{h}\right)\right) \cdot \mu_{T}^{h} d \Gamma=0 \quad \forall \mu^{h} \in W^{h} .
\end{array}\right.
$$

The existence of a solution to Problem (20) for any value of $r>0$ and $\mathscr{F} \geq 0$ can be obtained classically with Brouwer's fixed point theorem applied on $\mathscr{P}: W^{h} \rightarrow W^{h}$ defined by

$$
\left.\mathscr{P}\left(\xi^{h}\right)=P_{W^{h}}\left(\left(\xi_{N}^{h}\right)_{-}-r\left(u_{N}^{h}-g\right)\right) n_{0}+P_{B\left(0, \mathscr{F}\left(\xi_{N}^{h}\right)_{-}\right)}\left(\xi_{T}^{h}\right)-r u_{T}^{h}\right),
$$

where $u$ is the solution to the first equation of (20) and $P_{W^{h}}$ is the orthogonal projection on $W^{h}$ (the technique of proof can be found for instance in [30]). The uniqueness for both $r>0$ and $\mathscr{F} \geq 0$ small enough can be obtained as follows:

Proposition 7.1 The solution $\left(u^{h}, \xi^{h}\right)$ to Problem (20) is unique provided that $a(\cdot, \cdot)$ is coercive, the inf-sup condition (13) is satisfied and for $r>0$ and $\mathscr{F} \geq 0$ small enough.

Proof. Let $\left(u^{h, 1}, \xi^{h, 1}\right)$ and $\left(u^{h, 2}, \xi^{h, 2}\right)$ be two solutions to Problem (20). Denoting $\lambda_{N}^{h, 1}=\left(\xi_{N}^{h, 1}\right)_{-}$, $\lambda_{N}^{h, 2}=\left(\xi_{N}^{h, 2}\right)_{-}, \lambda_{T}^{h, 1}=P_{B\left(0, \mathscr{F}\left(\xi_{N}^{h}\right)_{-}\right)}\left(\xi_{T}^{h, 1}\right)$ and $\lambda_{T}^{h, 2}=P_{B\left(0, \mathscr{F}\left(\xi_{N}^{h}\right)_{-}\right)}\left(\xi_{T}^{h, 2}\right)$, Problem (20) implies for $i=1,2$ :

$$
\begin{gathered}
a\left(u^{h, i}, v^{h}\right)=l\left(v^{h}\right)+\int_{\Gamma_{C}} \lambda_{N}^{h, i} v_{N}^{h} d \Gamma+\int_{\Gamma_{C}} \lambda_{T}^{h, i} \cdot v_{T}^{h} d \Gamma \quad \forall v^{h} \in V^{h}, \\
\int_{\Gamma_{C}}\left(\xi^{h, i}+r u^{h, i}-\lambda^{h, i}\right) \cdot \mu^{h} d \Gamma=0 \quad \forall \mu^{h} \in W^{h} .
\end{gathered}
$$

In particular, this leads to $\xi^{h, i}=P_{W^{h}}\left(\lambda^{h, i}-r u^{h, i}\right)$ and thus the following estimate holds:

$$
\begin{aligned}
\left\|\xi^{h, 1}-\xi^{h, 2}\right\|_{0, \Gamma_{C}}^{2} & =\left\|P_{W^{h}}\left(\lambda^{h, 1}-r u^{h, 1}\right)-P_{W^{h}}\left(\lambda^{h, 1}-r u^{h, 1}\right)\right\|_{0, \Gamma_{C}}^{2} \\
& \leq\left\|\lambda^{h, 1}-r u^{h, 1}-\lambda^{h, 2}+r u^{h, 2}\right\|_{0, \Gamma_{C}}^{2} \\
& =\left\|\lambda^{h, 1}-\lambda^{h, 2}\right\|_{0, \Gamma_{C}}^{2}-2 r \int_{\Gamma_{C}}\left(\lambda^{h, 1}-\lambda^{h, 2}\right)\left(u^{h, 1}-u^{h, 2}\right) d \Gamma+r^{2}\left\|u^{h, 1}-u^{h, 2}\right\|_{0, \Gamma_{C}}^{2} \\
& =\left\|\lambda^{h, 1}-\lambda^{h, 2}\right\|_{0, \Gamma_{C}}^{2}-2 r a\left(u^{h, 1}-u^{h, 2}, u^{h, 1}-u^{h, 2}\right)+r^{2}\left\|u^{h, 1}-u^{h, 2}\right\|_{0, \Gamma_{C}}^{2} .
\end{aligned}
$$



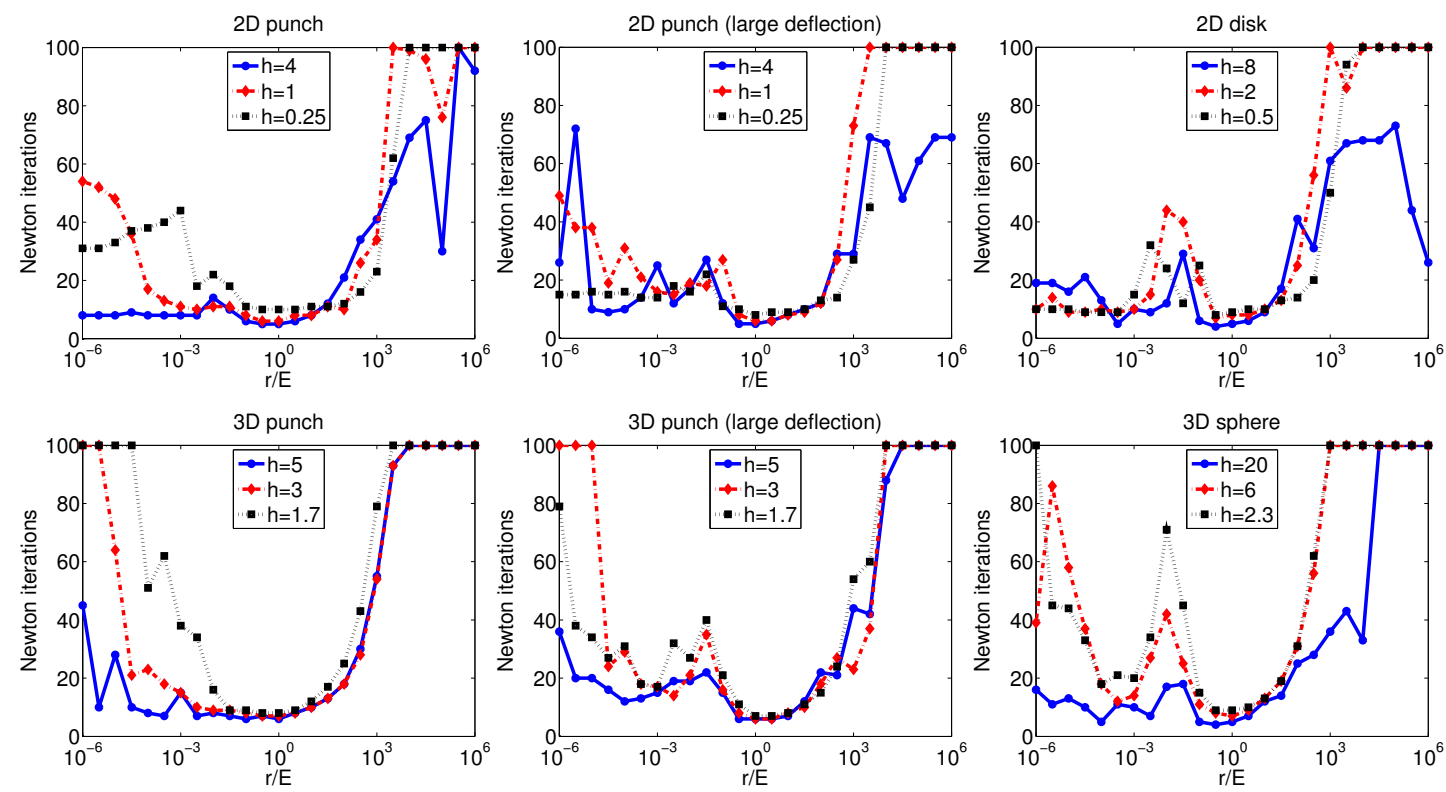

Figure 24: Number of iterations for the new Newton's method with integral frictionless contact approximation.

Due to the coercivity of $a(\cdot, \cdot)$ there exist $\alpha>0$ such that for $r>0$ sufficiently small one has

$$
-2 r a\left(u^{h, 1}-u^{h, 2}, u^{h, 1}-u^{h, 2}\right)+r^{2}\left\|u^{h, 1}-u^{h, 2}\right\|_{0, \Gamma_{C}}^{2} \leq-\alpha\left\|u^{h, 1}-u^{h, 2}\right\|_{0, \Gamma_{C}}^{2},
$$

which together with the inf-sup condition (13) leads to the existence of $\beta>0$ such that

$$
-2 r a\left(u^{h, 1}-u^{h, 2}, u^{h, 1}-u^{h, 2}\right)+r^{2}\left\|u^{h, 1}-u^{h, 2}\right\|_{0, \Gamma_{C}}^{2} \leq-\beta\left\|\lambda^{h, 1}-\lambda^{h, 2}\right\|_{0, \Gamma_{C}}^{2} .
$$

Moreover one has

$$
\left\|\lambda^{h, 1}-\lambda^{h, 2}\right\|_{0, \Gamma_{C}} \leq\left\|\xi^{h, 1}-\xi^{h, 2}\right\|_{0, \Gamma_{C}}+\left\|P_{B\left(0, \mathscr{F}\left(\xi_{N}^{h, 1}\right)_{-}\right)}\left(\xi_{T}^{h, 2}\right)-P_{B\left(0, \mathscr{F}\left(\xi_{N}^{h, 2}\right)_{-}\right)}\left(\xi_{T}^{h, 2}\right)\right\|_{0, \Gamma_{C}},
$$

and

$$
\left\|P_{B\left(0, \mathscr{F}\left(\xi_{N}^{h, 1}\right)_{-}\right)}\left(\xi_{T}^{h, 2}\right)-P_{B\left(0, \mathscr{F}\left(\xi_{N}^{h, 2}\right)_{-}\right)}\left(\xi_{T}^{h, 2}\right)\right\|_{0, \Gamma_{C}} \leq \mathscr{F}\left\|\xi_{N}^{h, 1}-\xi_{N}^{h, 2}\right\|_{0, \Gamma_{C}} .
$$

Thus, the following estimate holds for $r>0$ small enough:

$$
\left\|\xi^{h, 1}-\xi^{h, 2}\right\|_{0, \Gamma_{C}} \leq \sqrt{1-\beta}(1+\mathscr{F})\left\|\xi^{h, 1}-\xi^{h, 2}\right\|_{0, \Gamma_{C}},
$$

which leads to $\xi^{h, 1}=\xi^{h, 2}$ for $\mathscr{F}$ sufficiently small.

Note that since the solution to Problem (20) do depend on the parameter $r$, the uniqueness of the solution for an arbitrary $r>0$ is an open question. 

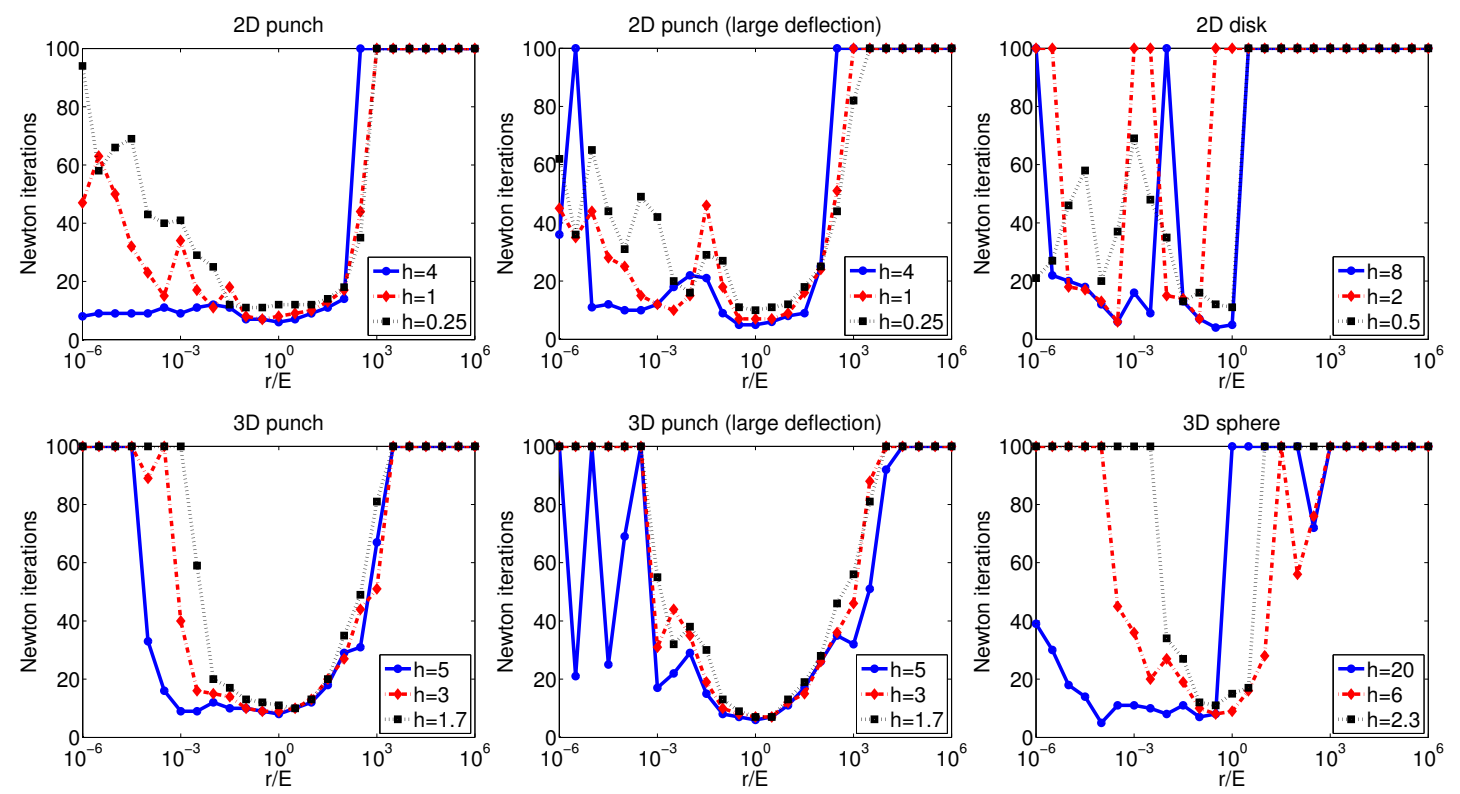

Figure 25: Number of iterations for the new Newton's method with integral contact with friction approximation.

The corresponding numerical experiments are presented in Fig. 24 and Fig. 25 for the contact without and with friction, respectively. Similarly to the Alart-Curnier approach, generalized Newton's method on the integral approximation leads to a less robust method with respect to the parameter $r$. When considering additionally De Saxcé's projection for the case with friction, the graphs of Fig. 26 show also an improvement of the convergence similarly to what was noted for the nodal approximation.

\section{Concluding remarks}

In this paper, some new generalized Newton's method for contact with friction problems are introduced and compared to existing strategies on some basic 2D and 3D situations. Two kinds of approximations of the contact and friction conditions are considered: a nodal and an integral one. The advantage of the integral approximation is the fact that it is without constraints. Consequently, the set of admissible stress is not to be described. This may be a crucial advantage for instance in the framework of fictitious domain or Xfem methods. However, this approximation leads to Newton's methods a little bit more sensitive to the value of the augmentation parameter compared to the ones build on a nodal approximation.

The new generalized Newton's methods based on augmented multipliers have a better behavior than the other tested methods, especially when using De Saxcé's projection. In particular it solves correctly the non-coercive cases. Of course, the method can be adapted to more complex contact situations such as two-body contact and large deformations. Despite the fact that it does not solves the non-coercive cases every time, non-symmetric Nitsche's method is also very promising and prevent the use of a multiplier which can be an advantage in some situations.

For all the methods, it is observed a slight increase of the number of Newton iterations when the mesh is refined. This variation indicates the non-scalability of the Newton's methods. However, this increase is quite small and, a priori, does not require a specific treatment. 

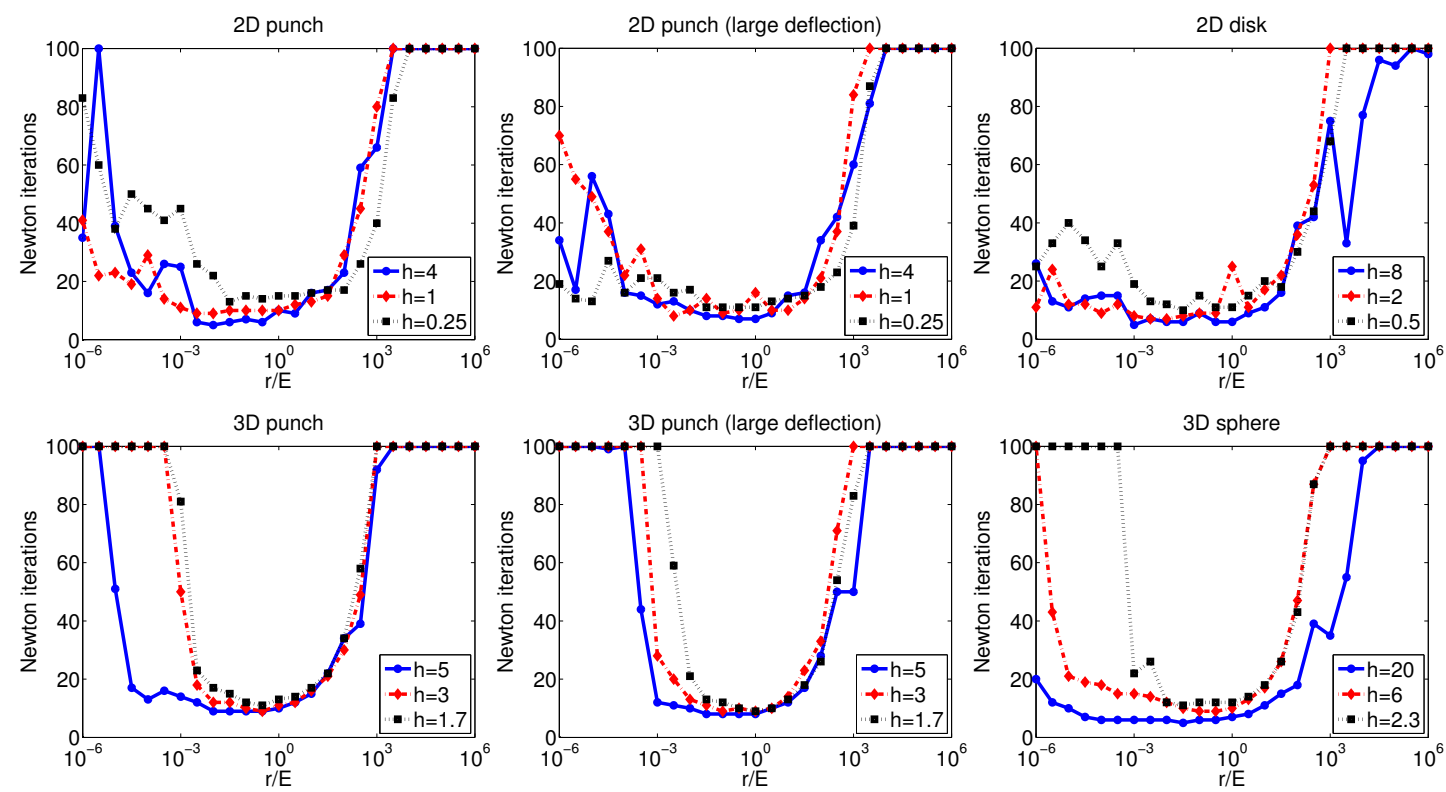

Figure 26: Number of iterations for the new Newton's method using De Saxcé projection and with integral contact with friction approximation.

\section{References}

[1] P. Alart. Méthode de Newton Généralisée en Mécanique du contact J. Math. Pures Appl., 76 (1997), 83-108.

[2] P. Alart, A. Curnier. A generalized Newton method for contact problems with friction $J$. Mech. Theor. Appl., 7:1 (1988), 67-82.

[3] P. Alart, A. Curnier. A mixed formulation for frictional contact problems prone to Newton like solution methods Comp. Meth. Appl. Mech. Engng., 92 (1991), 353-375.

[4] F. Ben Belgacem, Y. Renard. Hybrid finite element methods for Signorini's problem. Math. Comp., 72 (2003), 1117-1145.

[5] H. Ben Dhia, M. Zammali. Level-sets field, placement and velocity based formulations of contact-impact problems. Int. J. Numer. Meth. Engng., 69 (2007), 2711-2735.

[6] H. Ben Dhia, M. Zarroug. Hybrid frictional contact particles-in elements. Eur. J. Comp. Mech., 11 (2002), 417-430.

[7] P.G. Ciarlet. The finite element method for elliptic problems, Studies in Mathematics and its applications. North Holland Publishing, 1978.

[8] P. Chabrand, F. Dubois, M. Raous. Various numerical methods for solving unilateral contract problems with friction. Math. Comput. Model, 28:4-8 (1998), 97-108.

[9] F. Chouly, P. Hild. A Nitsche-base method for unilateral contact problems: numerical analysis. submitted

[10] F. Chouly, P. Hild, Y. Renard. Symmetric and non-symmetric variants of Nitsche's method for contact problems in elasticity: theory and numerical experiments submitted 
[11] P.W. Christensen, J.S. Pang. Frictional contact algorithms based on semismooth Newton methods, in: M. Fukushima, L. Qi (Eds.), Reformulation, Nonsmooth, Piecewise Smooth, Semismooth and Smoothing Methods, Kluwer Academic, Dordrecht, 1998, 81-116.

[12] P.W. Christensen. A semi-smooth Newton method for elasto-plastic contact problem. Int. J. Solids Struct. 39(2002), 2323-2341.

[13] G. DeSaxcé. The bipotential method: A constructive approach to design the complete contact law with friction and improved numerical algorithms Mathematical and Computer Modelling, 28:4-8(1998), 225-245.

[14] G. Duvaut, J.L. Lions. Les inéquations en mécanique et en physique. Dunod Paris, 1972.

[15] C. Eck, J. Jarušek. Existence results for the static contact problem with Coulomb friction. Mathematical Models and Methods in Applied Sciences, LV-8 (1998), 445-468.

[16] A. Ern, J.-L. Guermond. Éléments finis: théorie, applications, mise en ouvre. Mathématiques et Applications 36, SMAI, Springer-Verlag, 2002.

[17] Numerical methods for unilateral problems in solid mechanics in Handbook of Numerical Analysis, Volume IV, Part 2, P.G. Ciarlet and J. L. Lions, eds., North-Holland, Amsterdam, 1996, 313-485.

[18] P. Heintz, P. Hansbo. Stabilized Lagrange multiplier methods for bilateral elastic contact with friction, Comp. Meth. Appl. Mech. Eng., 195(2006), 4323-4333.

[19] P. HILD, Non-unique slipping in the Coulomb friction model in two-dimensional linear elasticity,

[20] P. HILD, Multiple solutions of stick and separation type in the Signorini model with Coulomb friction, Z. Angew. Math. Mech., 85 (2005), 673-680.

[21] P. HiLd, Y. RENARD, An improved a priori error analysis for finite element approximations of signorini's problem. Siam J. on Numer. Anal., 50:5 (2012), 2400-2419.

[22] S. Hber, B. Wohlmuth a primal-dual active set strategy for non-linear multibody contact problems. Comp. Meth. Appl. Mech. Engng., 194:27 (2005), 3147-3166.

[23] J. Jarušek. Contact problems with bounded friction. Coercive case. Czechoslovak Math. J., 33(1983), 237-261.

[24] Y. Kanno, J.A.C. Martins, A. Pinto da Costa. Three-dimensional quasi-static frictional contact by using second-order cone linear complementarity problem Int. J. Numer. Meth. Engng., 65(2006), 62-83.

[25] H. Khenous, J. Pommier, Y. Renard. Hybrid discretization of the Signorini problem with Coulomb friction, theoretical aspects and comparison of some numerical solvers. Applied Numerical Mathematics, 56/2 (2006),163-192.

[26] N. Kikuchi, J.T. Oden. Contact problems in elasticity, SIAM, 1988.

[27] K.C. Klarbring. Quadratic programs in frictionless contact problems. Int J Eng Sci, 24:7(1986), 1207-1217.

[28] K.C. Klarbring, J.-S. Pang. Existence of solutions to discrete semicoercive frictional contact problems. SIA J. Optim., 8(1998), 414-442.

[29] K. Kunish, G. Stadler. Generalized Newton methods for the 2D-Signorini contact problem with friction in function space. Mathematical Modelling and Numerical Analysis, 39:4 (2005), $827-854$. 
[30] P. Laborde, Y. Renard. Fixed point strategies for elastostatic frictional contact problems. Math. Meth. Appl. Sci., 31(2008), 415-441.

[31] T.A. Laursen, J.C. Simo. Algorithm symmetrization of Coulomb frictional problems using augmented Lagrangians. Comp. Meth. Appl. Mech. Engng, 108(1993), 133-146.

[32] A. Leontiev, W. Huacasi, J. Herskovits. An optimization technique for the solution of the Signorini problem using the boundary element method. Struct Multidisc Optim, 24 (2002), $72-77$.

[33] J. Nitsche, Über ein Variationsprinzip zur Lösung von Firichlet-Problement bei Verwendung von Teilräumen, die keinen Randdbedingungen unterworfen sind, Abhandlungen aus dem Mathematischen Seminar der Universität Hamburg, 36 (1971), 9-15.

[34] L. Qi, J. Sun. A nonsmooth version of Newton's method. Mathematical Programming, 58 (1993), 353-367.

[35] M. Raous, S. Barbarin. Preconditionned conjugate gradient method for a unilateral problem with friction. In Contact Mechanics, A. Curnier Editor, PPUR, Lausane 1992, 423-432.

[36] Y. Renard, A uniqueness criterion for the Signorini problem with Coulomb friction, SIAM J. Math. Anal., 38 (2006), 452-467.

[37] R.T. Rockafellar, R. J-B. Wets. Variational Analysis. Springer-Verlag, 1998.

[38] G. Tanoh, Y. Renard, D. Noll. Computational experience with an interior point algorithm for large scale contact problems. Technical Report 04.22, MIP, Université Paul Sabatier, Toulouse, http://www.optimization-online.org/DB_HTML/2004/12/1012.html, 2004.

[39] M. Siavelis, P. Massin, M.L.E. Guiton, S. Mazet, N. Moës. Robust implementation of contact under friction and large sliding with the extended finite element method Eur. J. Comp. Mech., 19:1-2-3(2010), 189-203.

[40] J.C. Simo, T.A. Laursen. An augmented Lagrangian treatment of contact problems involving friction. Comput. \& Structures, 42(1992), 97-116.

[41] G. Stadler. Infinite-dimensional semi-smooth Newton and augmented Lagrangian methods for friction and contact problems in elasticity. PhD thesis (2004).

[42] M. Ulbrich. Semismooth newton methods for variational inequalities and constrained optimization problems in function spaces. MOS-SIAM Series on Optimization, SIAM, Philadelphia, 2011.

[43] P. Wriggers, G. Zavarise. A formulation for frictionless contact problems using a weak form introduced by Nitsche. Comput. Mech. 41 (2008), 407-420.

[44] G. Zavarise, L. De Lorenzis, and R.L. Taylor. A non-consistent start-up procedure for contact problems with large load-steps. Comput. Methods Appl. Mech. Engrg., 205-208(2012), 91109. 